Document downloaded from:

http://hdl.handle.net/10251/147684

This paper must be cited as:

Parvulescu, VI.; García Gómez, H. (2018). Heterogeneous catalysis based on supramolecular association. Catalysis Science \& Technology. 8(19):4834-4857. https://doi.org/10.1039/c8cy01295d

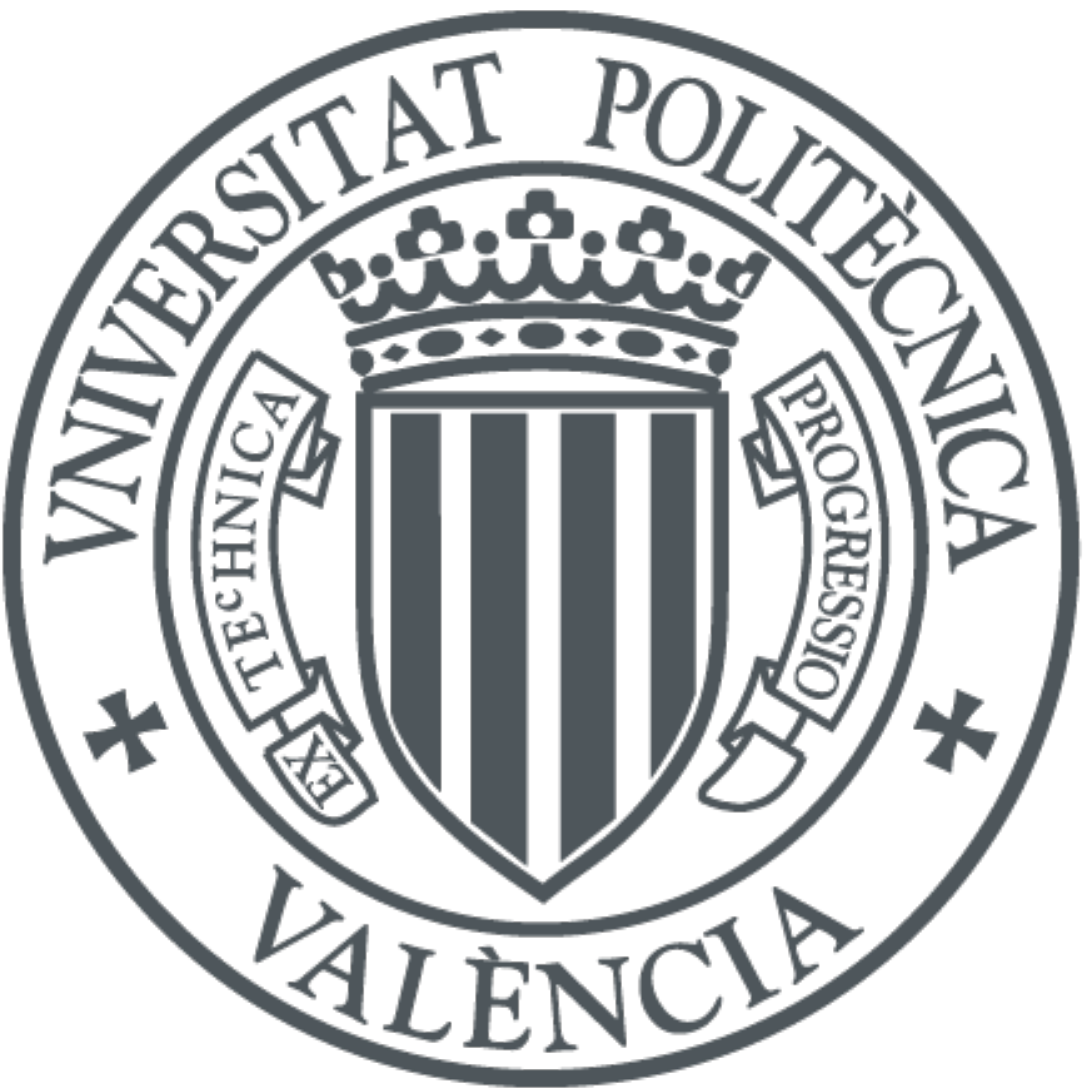

The final publication is available at

https://doi.org/10.1039/c8cy01295d

Copyright The Royal Society of Chemistry

Additional Information 


\section{Heterogeneous catalysis based on the supramolecular association}

Vasile I. Parvulescu ${ }^{\mathrm{a}}$ and Hermenegildo García ${ }^{\mathrm{b}}$

${ }^{a}$ Department of Organic Chemistry, Biochemistry and Catalysis ,Faculty of Chemistry, University of Bucharest, Bdul Regina Elisabeta 4-12, Bucharest 030016, Romania. E-mail: vasile.parvulescu@chimie.unibuc.ro

${ }^{b}$ Instituto Universitario de Tecnología Química Consejo Superior de Investigaciones Científicas-Universitat Politecnica de Valencia, Universitat Poliecnica de Valencia, Av. De los Naranjos s/n, 46022 Valencia, Spain. E-mail: hgarcia@qim.upv.es

\footnotetext{
Abstract. Heterogeneous catalysis is based mostly in materials that built with strong covalent bonds. However, supramolecular aggregation in which individual components selfassemble due to non-covalent interactions to create a larger entity offers also considerable potential for the preparation of materials with application in catalysis. The present article provides a perspective on the use of supramolecular aggregation for the development of heterogeneous catalysts. One of the main advantages of this approach is that the preparation procedure based on spontaneous self-assembly is frequently simpler than those that require the formation of covalent bonds. The emphasis in this article has been made on the use in the preparation of heterogeneous catalyst of carbon materials, particularly graphenes and carbon nantotubes, but also dendrimers and organic capsules. Examples of hybrid organic-inorganic materials such as mesoporous organosilicas, metal-organic frameworks and heteropolyacids are also briefly described. The purpose is to illustrate the breadth of the field and the diverse array of possibilities already developed to apply the concepts of supramolecular association in heterogeneous catalysis.
} 


\section{Introduction.}

Supramolecular chemistry aims at the design, synthesis and understanding of the assembly of a discrete number of molecular entities into a larger ensembles. By association into larger aggregates, new entities with larger size and shape are produced. Electronic properties and functionality of the assembly may also be different from those of individual components $[1,2]$. The spatial organization of the molecular units is controlled by intermolecular forces such as hydrogen bonding, $\pi-\pi$ stacking and dispersion forces that are weaker than covalent bonding. Nano-segregation, and steric requirements of the peripheral building blocks also influence the assemble of components.[3] Further, the concentration, the nature of the solvent or the presence of additive may also induce a supramolecular self-organisation known as a J-stacking [4, 5]. The differences between the $\mathrm{H}$ - and J-stacking pattern in dyes can be easily evidenced from the spectral shifts in different media (Figure 1). The observed bathrochromically shifted J-bands and hypsochromically shifted H-bands of the aggregates have been explained in terms of coupling of transition moments of the constituent dye molecules. 
A

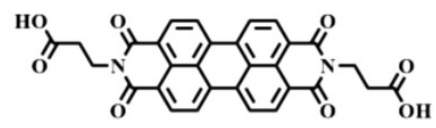

H-PDI molecule

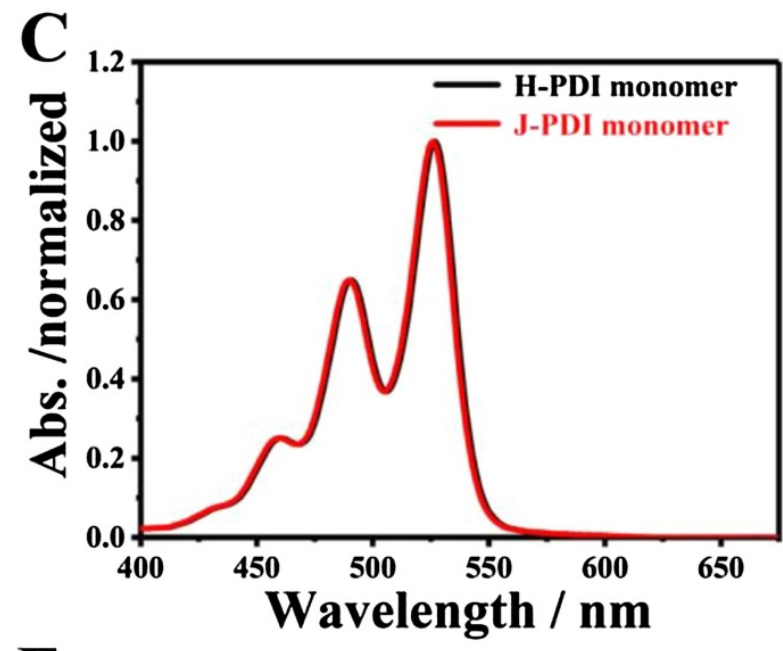

$\mathbf{E}$

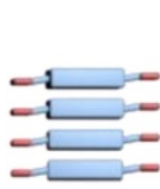

Rotationally displace

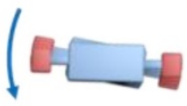

B

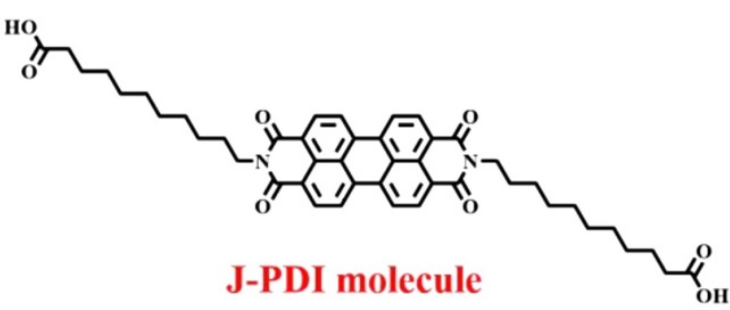

D

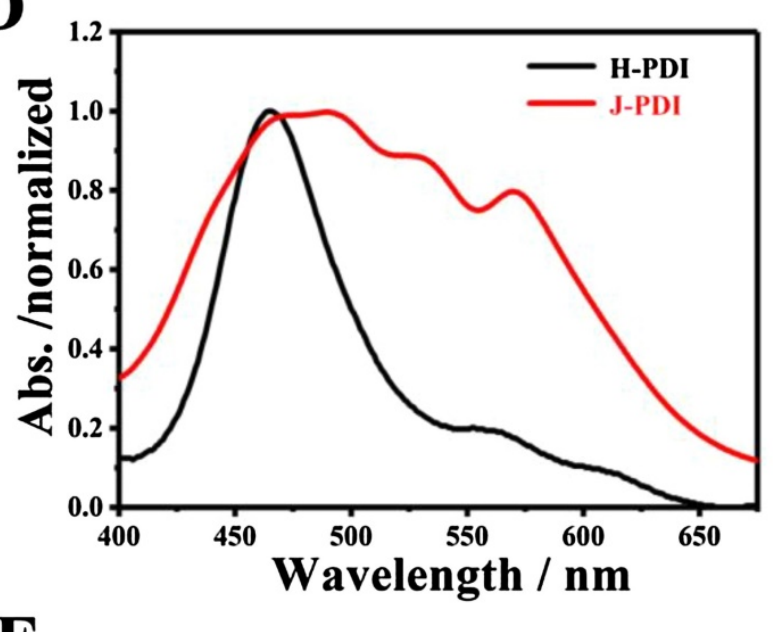

F

\section{Laterally displace Rotationally displace}

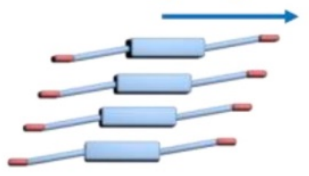

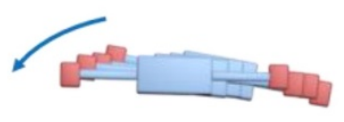

J-aggregate

Figure 1. (A, B) Molecular structures of H-PDI (A) and J-PDI (B). (C) UV-vis spectra of H-PDI monomer and J-PDI monomer. (C) UV-vis spectra of H-PDI and J-PDI nanostructures. (E, F) Supramolecular packing model of H-aggregates (E) and J-aggregates (F). With permission from ref. [6].

The different interactions occurring in the solution may lead to the formation of various assemblies with different dimensions that could even be translated in the solid state. The protonation of the components may, on the other hand, result in the formation of entirely different 
supramolecular structures, due to the formation of strong hydrogen bonding.[7] Coordination of basic solvents to the supramolecular aggregate and hydrogen bonding with protic solvents also influence the structural properties of these entities [7].

Formation of supramolecular aggregates can result in a certain degree of electronic coupling among the molecular components. However, the energy of this electronic coupling is frequently small compared to common covalent bond energy values.

Supramolecular aggregates have attracted the interests in many fields, especially in biochemistry and life sciences, but also in nanoscience and nanotechnology, surface and material science, etc. [8-12]. One of the most important distinctive features that can exhibit supramolecular aggregates is a new functionality not present in any of the individual component. This new functionality derives from the interplay of the components of the new structure arising from the aggregation. The present article gives an overview of the application of supramolecular aggregation for the development of heterogeneous catalysts. Emphasis is made on those examples in which higher selectivity and/or improved activity as consequence of the association of the components in the supramolecular entity $[13,14]$. The first section is focused on the synthesis of supramolecular structures involving graphene and carbon nanotubes for their application as heterogeneous catalysts. Comparison of the performance of these supramolecular systems with that of the homologous ones lacking the supramolecular association will be provided, when available. The purpose is to highlight the advantages of the supramolecular systems and the origin of this superior activity. The potential of the use of supramolecular aggregates with carbon nanotubes for the electrochemical activation of biocatalysts will also be presented. The last sections include a brief overview on the use of supramolecular assemblies using cyclodextrins, 
rotaxanes, organic polyaromatic compounds and organic-inorganic hybrids to build supramolecular entities with potential application in catalysis.

\section{Synthesis of solid catalysts following a supramolecular approach}

\section{Self-assembling protocols}

Efficient electrocatalysts for the oxygen reduction reaction (ORR) and methanol electrooxidation have been obtained following a supramolecular approach. With this aim, nitrogen-doped three-dimensional (3D) porous graphene frameworks were fabricated starting from graphene oxide as building block and a supramolecular aggregate of self-assembled melamine and cyanuric acid (Figure 2). Such aggregates act not only a "spacer” to suppress the re-stacking of graphene nanosheets, but also as a sacrificial pore-forming template and as nitrogen source leading to $\mathrm{N}$-doping of the resulting reduced graphene oxide [15]. The porous material possesses a specific 3D architecture with enhanced ORR (the positive half-wave potential is only $\sim 43 \mathrm{mV}$ ) compared to commercial Pt/C, and high methanol poisoning tolerance. 


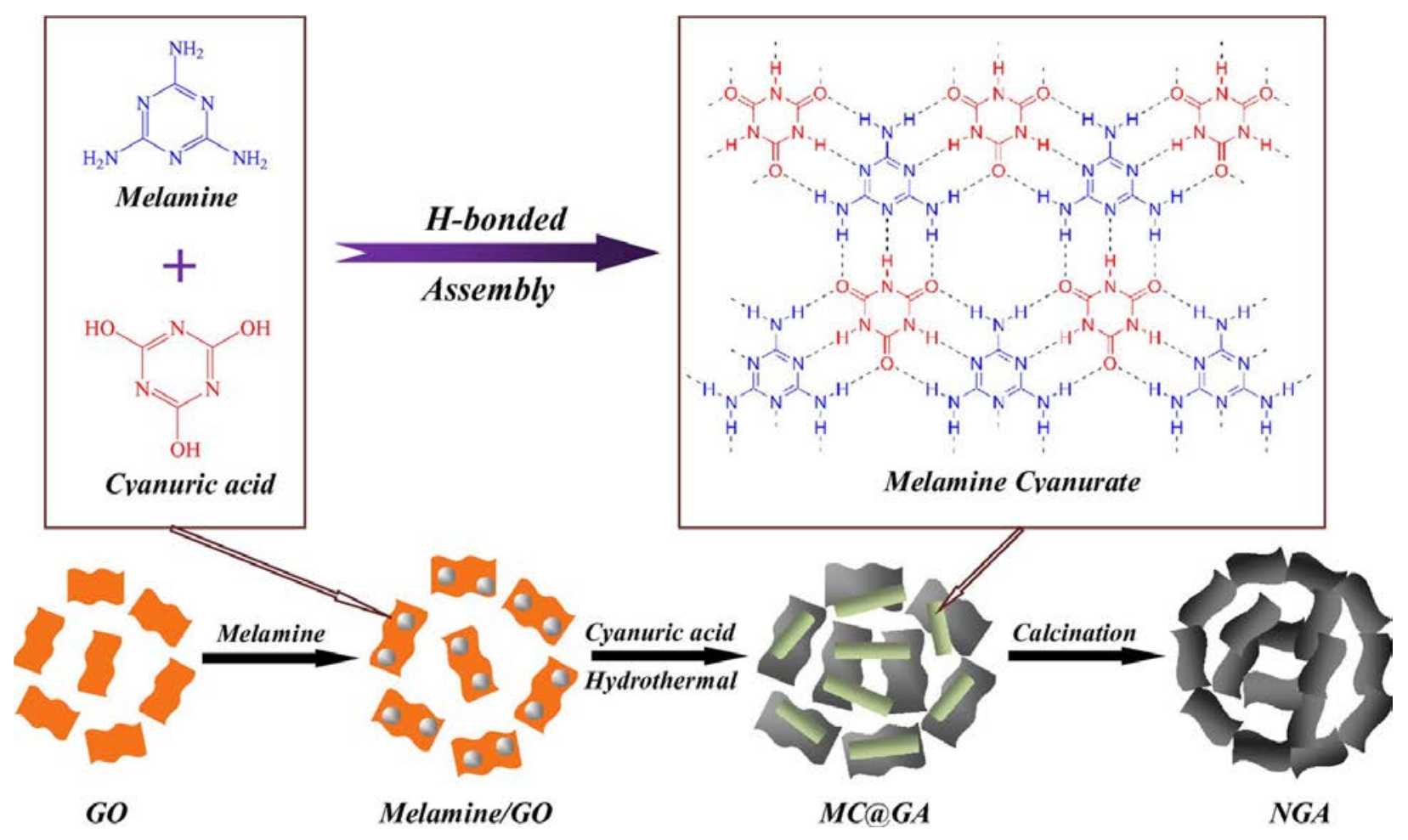

Figure 2. Synthetic procedure of $\mathrm{N}$-doped 3D porous graphene frameworks prepared via a supramolecular approach. GO: Graphene oxide. With permission from ref. [15].

3D g- $\mathrm{C}_{3} \mathrm{~N}_{4}$ (g meaning graphitic) aggregates have also been prepared following a supramolecular approach [16]. This material is composed of hollow bubbles developed by hydrothermally treatment of a supramolecular precursor in the presence of Pluronic P-123 as soft template. During the annealing of the intermediate, long balloons were first formed and then transformed into hollow bubbles. A $2 \mathrm{D}$ g- $\mathrm{C}_{3} \mathrm{~N}_{4}$ material was prepared by using another supramolecular route from a precursor obtained by $\mathrm{HCl}$-assisted aqueous solution hydrothermal process of melamine [16]. These catalysts are able to generate upon visible light irradiation $\bullet^{-\mathrm{O}_{2}}$ and $\bullet \mathrm{OH}$ active species that are efficient degrading tetracycline [16]. The same material can also generate photocatalytically hydrogen [16]. 


\section{Synthesis based on host-guest interactions}

There are synthesis protocols of supramolecular assemblies based on the formation of host-guest encapsulation and $\mathrm{CH}-\pi$ interactions. These procedures have successfully applied to the synthesis of supramolecular systems incorporating metal nanoparticles [17]. In one of these reports, end-toend assembly of gold-nanorods was achieved based on hydrogen bonding of organic ligands with terminal carboxylic and/or amino groups. Alternatively, end-to-end assembly of the gold nanorods was also accomplished using metal complexes capable of metal-metal interactions (Figure 3) [18]. Importantly, this approach allows to construct reversible hybrid materials, which can be useful for the recycling and recovering of these colloids.

(a)

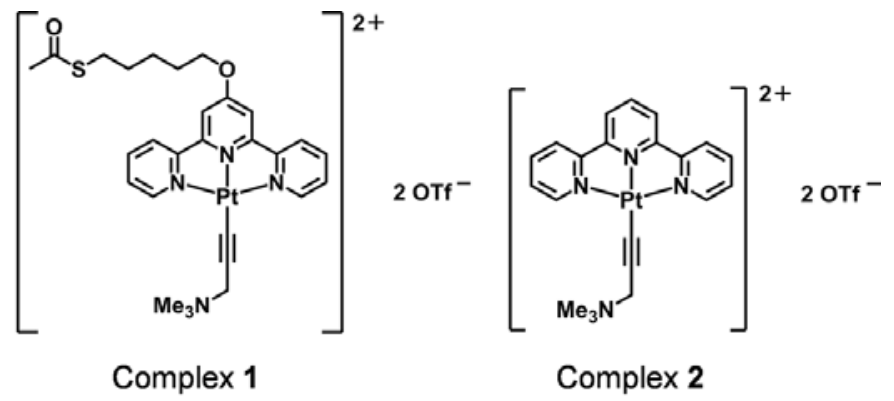

(b)

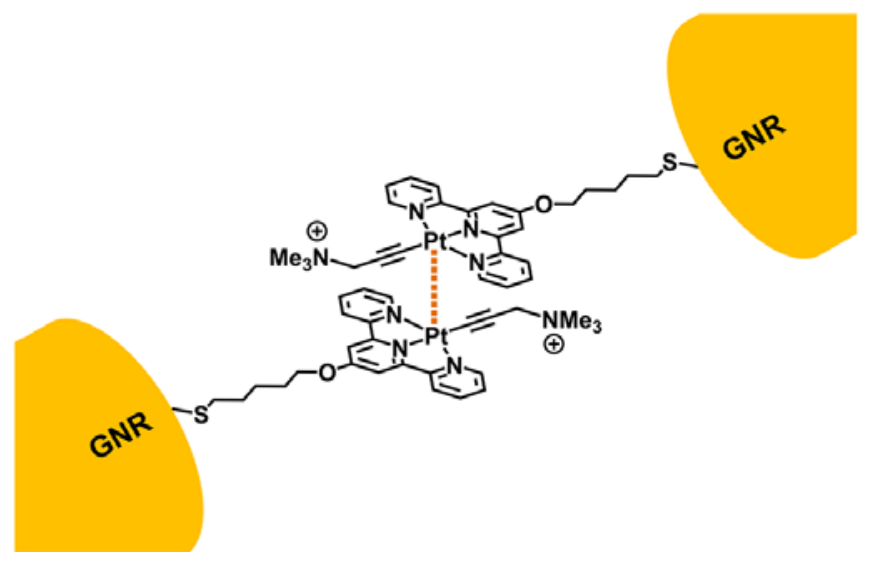


Figure 3. (a) Chemical structures of two Pt complexes. (b) Schematic diagram of the end-to-end assembly of gold nanorods induced by $\mathrm{Pt} \cdots \mathrm{Pt}$ and $\pi-\pi$ stacking interactions. With permission from [18].

Host-guest interactions also allow gel formation. Gels represent another class of soft materials linked by non-covalent interactions with application in catalysis [19]. Multi-functional metallacage-cored supramolecular gel was prepared by orthogonal metal-coordination and hostguest interactions. The key component is 21-crown-7 ether having a bis-4-pyridyl phenanthroline ligand to bind with a platinum salt. Crosslinking is achieved by addition of a bisammonium linker that interpenetrates the crown ether, while pyridyl units are bound to platinum metal forming a supramolecular polymer network via two types of interactions. Figure 4 presents the three components and the type of interactions resulting in a supramolecular gel. The same approach may provide chiral metallogels [20]. 


\section{(a)}

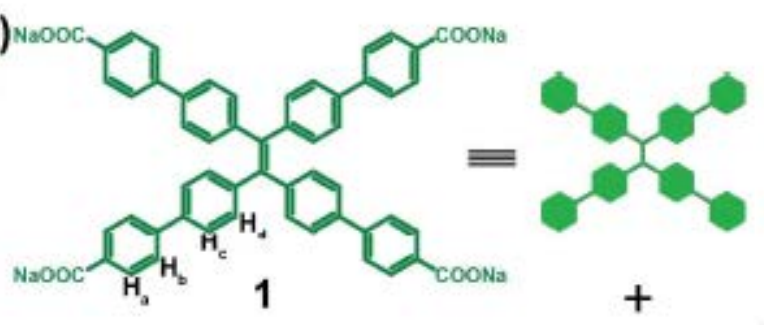

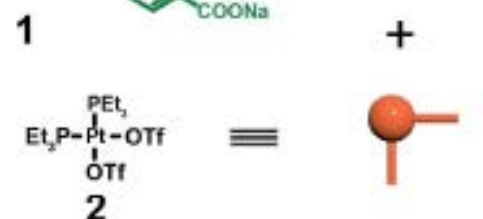

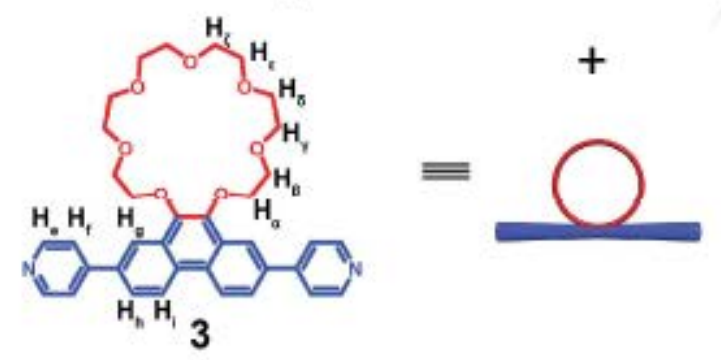
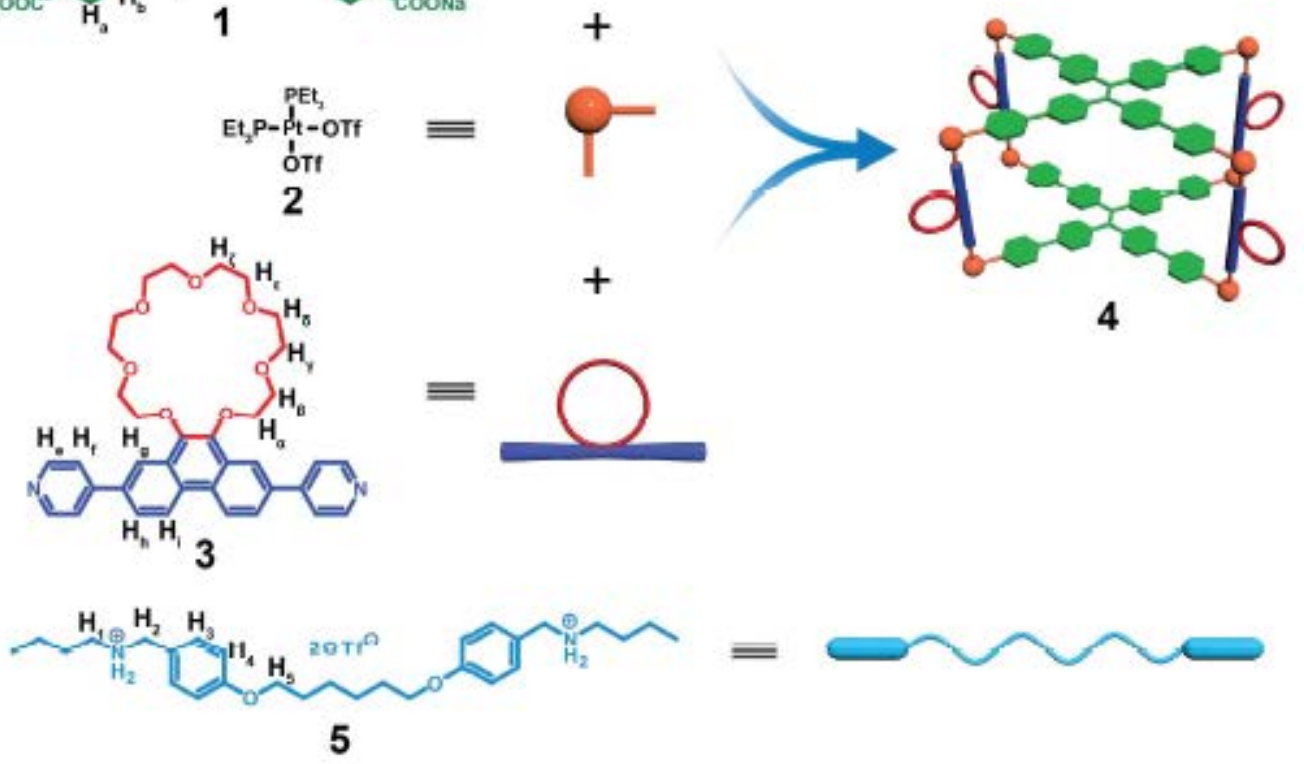

(b)

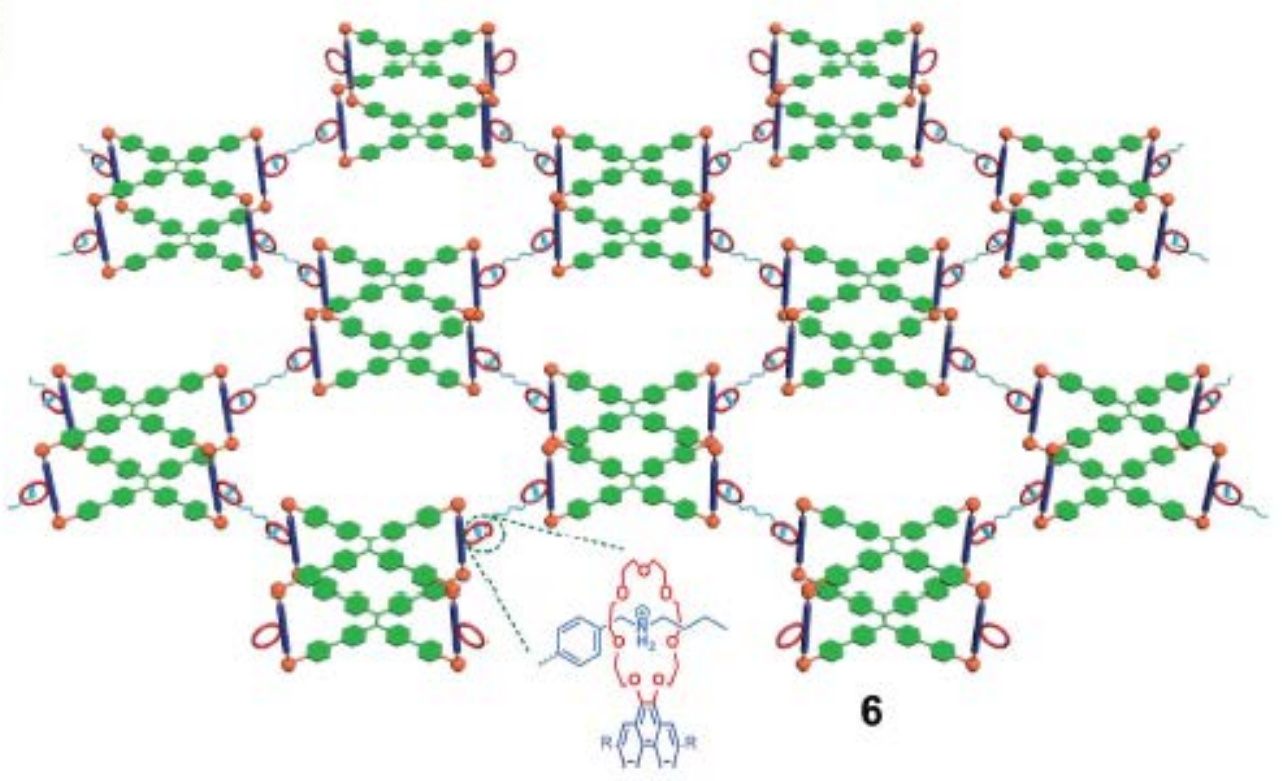

Figure 4. (a) Self-assembly of 21C7-functionalized metallacage and (b11) representation of the formation of a cross-linked supramolecular polymer network 6 from metallacage 4 and bisammonium salt $\mathbf{5}$. With permission from [19]. 


\section{Supramolecular catalysis with 2D graphene}

Adsorption of organic molecules on surfaces is one of the most general methodologies to modify the properties of the material. However, strategies for obtaining well-ordered adlayers with tailored functionalities based on adsorption still remain a key issue in nanotechnology.[21] The simplicity of spontaneous self-assembly through noncovalent adsorption of planar organic building blocks on graphene surfaces (graphene, carbon nanotubes, etc) is among the main reasons why this strategy has attracted considerable interest. Adsorption could lead to the modification of graphene properties.[21] The noncovalent functionalization of graphene offers the possibility of introducing functionality, while maintaining the integrity of the $\mathrm{sp}^{2}$-hybridized carbon network.[22] It should be, however, commented that the electronic properties of the substrate, adsorption capability and its surface availability are altered after the association respect to the pristine substrate. The degree of property variation is directly related to the surface coverage and strength of the interaction. In this regard, some of the initial advantages of the $\mathrm{sp}^{2}$ network integrity could be lost due to the formation of these supramolecular aggregates.

Polycyclic aromatic hydrocarbons are a class of compounds consisting of more than two benzene rings fused in a linear, angular, or clustered arrangement. Sorption of such molecules (naphthalene, pyrene, anthracene, fullerene, etc) onto structures incorporating aromatic entities is controlled by a complex mechanism dominated by $\pi-\pi$ interactions. Typically these polycyclic aromatic hydrocarbons form strong association complexes with materials having extended $\pi$ orbitals, like graphene and carbon nanotubes. This interaction is very strong, for instance, in the case of pyrene and derivatives. For this reason, having a molecule with a pyrenyl handle is a general methodology to adhere non-covalently functional groups to graphene and related materials 
[23]. Besides these 2D, 3D building blocks like Janus tectons may also afford such arrangements $[21,24]$.

It should be, however, commented that the presence of polar functionalities (such as carboxyl or carbonyl groups present in defective graphenes) may affect negatively to the association with condensed polycyclic aromatic entities based on $\pi-\pi$ interactions [25]. Surface area and morphology are other factors controlling the sorption.[26] It seems, however, that the interaction with extended aromatic surfaces (graphene, carbon nanotubes, graphite) can in some cases be maintained in a wide range of $\mathrm{pH}$ values when the aromatic hydrocarbon has no substituents. Thus, varying the $\mathrm{pH}$ from 2 to 11 did not affect the adsorption of naphthalene. However, in defective materials such as CNTs or graphene having oxygenated functional groups, deprotonation of the $-\mathrm{COOH}$ and $-\mathrm{OH}$ groups can increase electron density on the substrate. This increase in electron density can promote the $\pi$-electron-donor ability of the graphene surface, therefore enhancing $\pi$ - $\pi$ electron-donor-acceptor of functionalized $\pi$-electron acceptors, such as 1,3-dinitrobenzene or 1,3,5-trinitrobenzene.[27] The reverse should apply for the formation of charge-transfer association complexes with $\pi$-electron donor molecules. However, because different types of association forces, including hydrophobic interactions, $\pi$ - $\pi$ bonds, electrostatic interactions, and hydrogen bonds, sometimes with contradictory requirements, may act simultaneously, the prediction of the adsorption strength of organic molecules on CNTs/graphene is not straightforward. Thus, different models are needed to predict small organic molecule-CNT interaction depending on the prevalent type of operating intermolecular forces [26].

It is important to remind that although the energy of noncovalent interactions is normally much lower than those typical for covalent bonds, but when many of these interactions spread over large surfaces they can rival some covalent bonds. DFT calculations have provided rationalization 
for the observed trend of the increase of the binding energy with the increase in the number of rings in the polycyclic aromatic ring [28]. For appropriate ring sizes, the strength of noncovalent interactions allows the supramolecular system to be reversible, or kinetically labile, and, thus, selfrepairing. With graphenic materials, the $\pi-\pi$ or $\mathrm{CH}-\pi$ interactions typically exhibit dissociation energies lower than $50 \mathrm{~kJ} \mathrm{~mol}^{-1}$. In this case, the association forces are dominated by Londondispersion forces or van der Waals interactions [22].

As commented earlier, the selection of 2D graphenes and related materials as platforms for developing supramolecular catalysts is frequently based on their ability to form strong association complexes with polycyclic aromatic compounds. This strong adsorption can be advantageously exploited to be used as handles to develop supramolecular catalysts [29]. Molecules having intrinsic catalytic activity and encompassing in their structure condensed polycyclic subunits can interact through $\pi-\pi$ interaction with substrates. These $\pi-\pi$ interactions may alter the electronic properties of the substrate and, therefore, they have implications in catalysis, modifying the kinetics of the reactions in which these molecular catalysts are involved [29]. On the other hand, non-covalent interactions of these condensed polycyclic aromatic subunits can be conveniently used as a general approach for immobilization of active catalytic moiety onto the surface of graphene derivatives. This provides an easy, yet extremely effective, way to support catalysts and facilitate their recycling [29].

Using 2D graphene as platform to anchor molecular complexes, it has been found that the behavior of the supramolecular catalyst has certain specificity versus that of the molecular, unattached complex. These differences are derived from the ability of a peripheral $\pi$-stacking subunit, like pyrenyl, to establish non-covalent interactions with aromatic substrates or to attach strongly the metallic complex to the graphene sheets forming a supramolecular catalyst [30]. For 
analogous reactions involving non aromatic substrates or with related catalysts lacking polyaromatic tags such distinctive features do not exist. In the presence of an external $\pi$-stacking additive, the reaction of aromatic substrates follows a zero-order dependence on substrate concentration that is a consequence of the catalyst saturation. Non-aromatic substrates on the same catalysts exhibit second-order dependence. It was also found that the stability of the catalyst depends on the number of tags, catalysts with two tags exhibiting very good stability.

Examples of such catalytic reactions include hydrodefluorination. $\mathrm{C}-\mathrm{F}$ is among the most unreactive functionalities in chemistry and, therefore, its disruption requires harsh conditions and active enough catalysts. Interestingly, this reaction has been quantitatively achieved by a supramolecular catalyst consisting of a mixture of palladium and ruthenium complexes with a pyrene-NHC ligand (NHC: N-heterocyclic carbene) grafted on the surface of reduced-graphene oxide by $\pi$-stacking interactions (Figure 5) .[31] These interactions between pyrene and reduced graphene oxide are strong enough to allow the recycling of the catalyst in consecutive runs, exhibiting essentially the same performance.

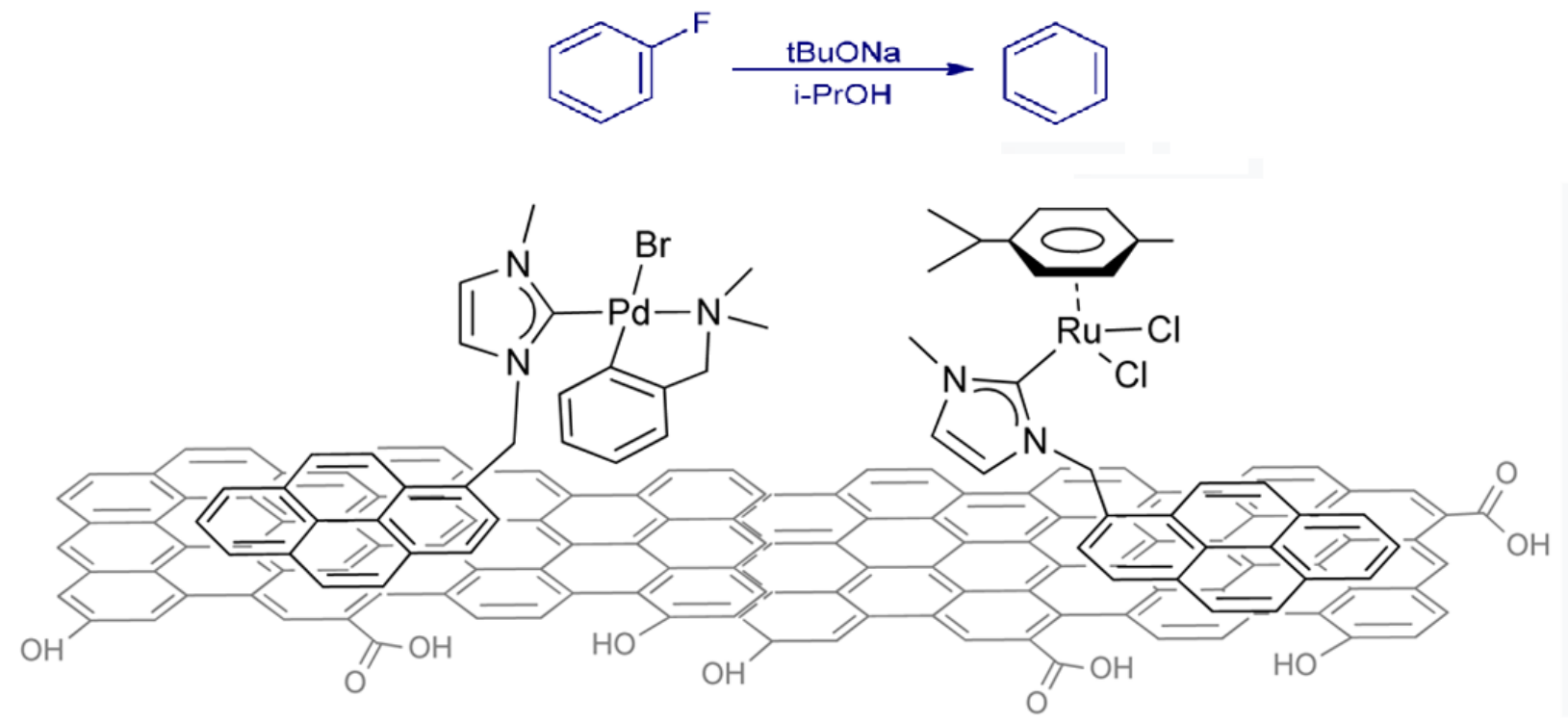


Figure 5. Hydrodefluorination of a series of fluoroarenes catalyzed by palladium and ruthenium complexes with pyrene-tagged NHC ligands immobilized onto reduced graphene oxide. Adapted from ref. [31]).

Following the same concept, reduced graphene oxide immobilized complexes with monoand bidentate pyrene-containing ligands like those presented in Figure 6 demonstrated efficiency and stability for $\beta$-alkylation of 1-phenylethanol with primary alcohols and hydrogenation of ketones [30].
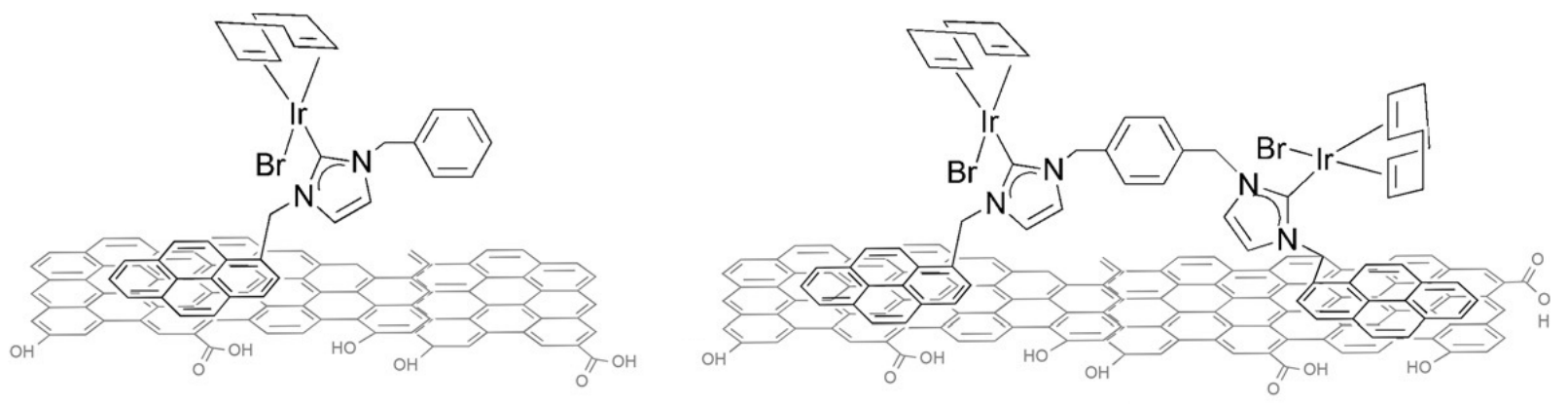

Figure 6. Mono- and bidentate pyrene-containing NHC ligands used to form supramolecular entities with reduced graphene oxide. Ir complexes are formed by binding through the NHC moieties. These Ir complexes exhibit catalytic activity for $\beta$-alkylation of 1 -phenylethanol with primary alcohols and hydrogenation of ketones. With permission from ref. [30].

The same catalysts exhibited very high activity for the hydrogenation of alkenes and nitro groups using molecular hydrogen and oxidant-free dehydrogenation of alcohols to ketones [32]. 
Recycling experiments carried out in toluene showed no decay in the performance of the material upon reuse.

Magnetic carbon-coated nanoparticles (MNPs) take also the advantage of the graphenelike shell offering the same possibility for non-covalent catalyst attachment by $\pi-\pi$ stacking with pyrene-tagged complexes, as previously commented [33, 34]. Taking this model, grafting pyrenyltagged dendritic structures, like Pd-phosphine catalysts, onto Co/C MNPs by $\pi-\pi$ stacking afforded high activity and recyclability in Suzuki reactions for the preparation of various biaryls (Scheme 1) [34]. Magnetic support enables stirring and recovery of the catalyst by applying an external magnetic field, which avoids the need for precipitation or filtration steps. Attaching a dendritic ligand onto the MNP surface allows up to five times higher loading of catalyst, resulting in an increased productivity per gram of catalyst. The leaching of the catalyst was in the specification limits for metal impurities in the pharmaceutical industry ( $<5 \mathrm{ppm} \mathrm{Pd})$.
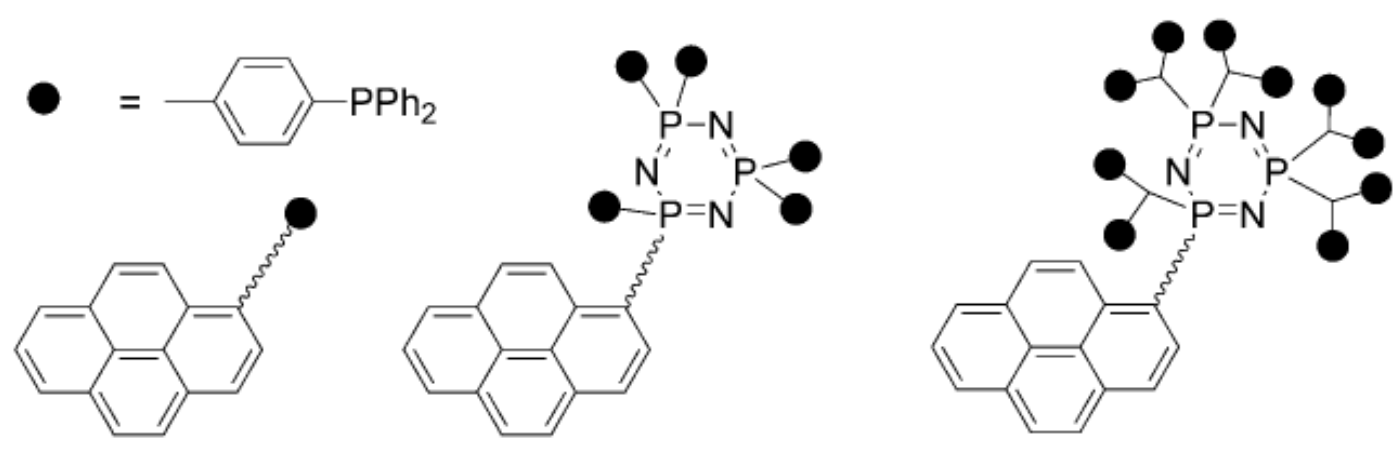

Scheme 1. Structures of monomeric and dendritic phosphine ligands that have been attached to reduced graphene oxide. With permission from ref. [34]. 
Non-covalent surface binding methods using strong van der Waals $\pi-\pi$ interactions between polyaromatic hydrocarbons and graphitized carbon surfaces offer also a convenient way for catalyst immobilization useful in electrocatalysis. One of these examples deals with the electrocatalytic $\mathrm{CO}_{2}$ reduction to formate using an Ir pincer dihydride complex attached through a pyrenyl handle to carbon nanotubes. This supported Ir pincer complex is present in the gas diffusion layer on top of the electrode (Figure 7). The electrocatalytic gas-liquid $\mathrm{CO}_{2}$ reduction occurs with notable rates, turnover numbers, and surface stability [34].

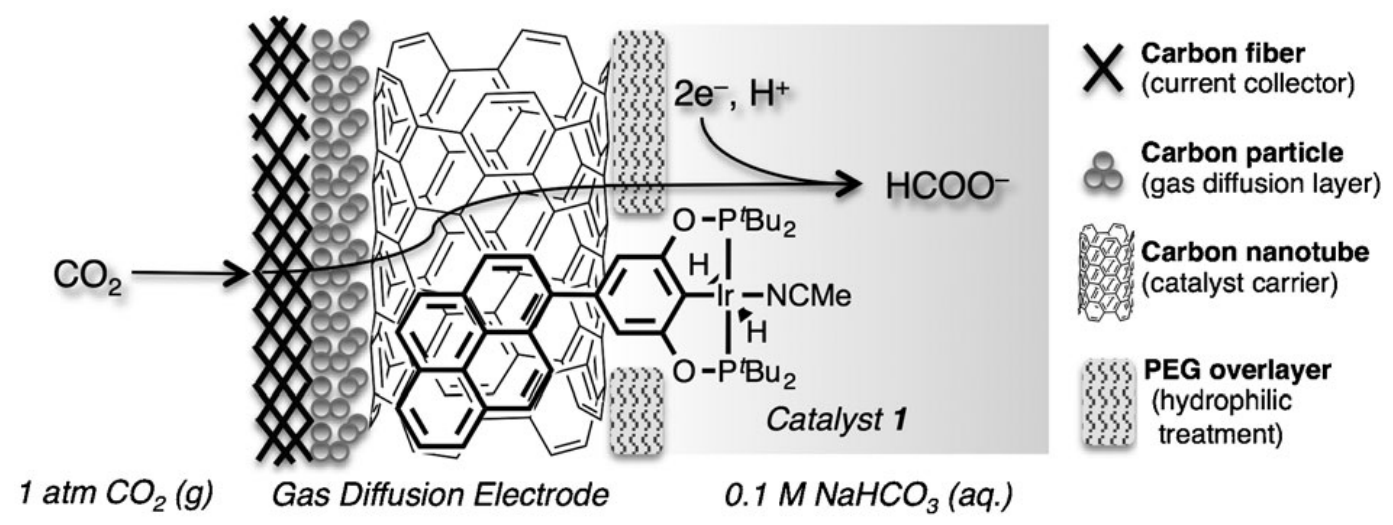

Figure 7. Illustration of a CNT-coated gas diffusion electrode with surface-bound Ir pincer dihydride catalyst through the pyrene-graphene $\pi-\pi$ stacking to promote the electrocatalytic reduction of $\mathrm{CO}_{2}$ to formate. Adapted from ref. [34].

In addition to the non-covalent attachment of the catalytic sites, supramolecular association of planar aromatic compounds on graphene can also play a role in the product formation. As an example, the synthesis of meso-aryl porphyrins and expanded porphyrins has been reported to be catalyzed by aryl sulfonic acid units covalently attached to the graphene sheet in dichloromethane and o-dichlorobenzene (Figure 8) [35]. The high catalytic activity at room temperature and high 
turnover number in the cross-condensation of dipyrromethane with benzaldehyde was primarily due to the catalysis by the sulfonic acid groups on the graphene. Besides acidity, the performance of the heterogeneous carbocatalyst was also dictated by the supramolecular $\pi-\pi$ interactions between the porphyrinic products and the basal graphene sheet. This proposal has been supported by observing a red shift in the Soret bands and by the quenching of the fluorescence intensity of the otherwise strongly fluorescent products.
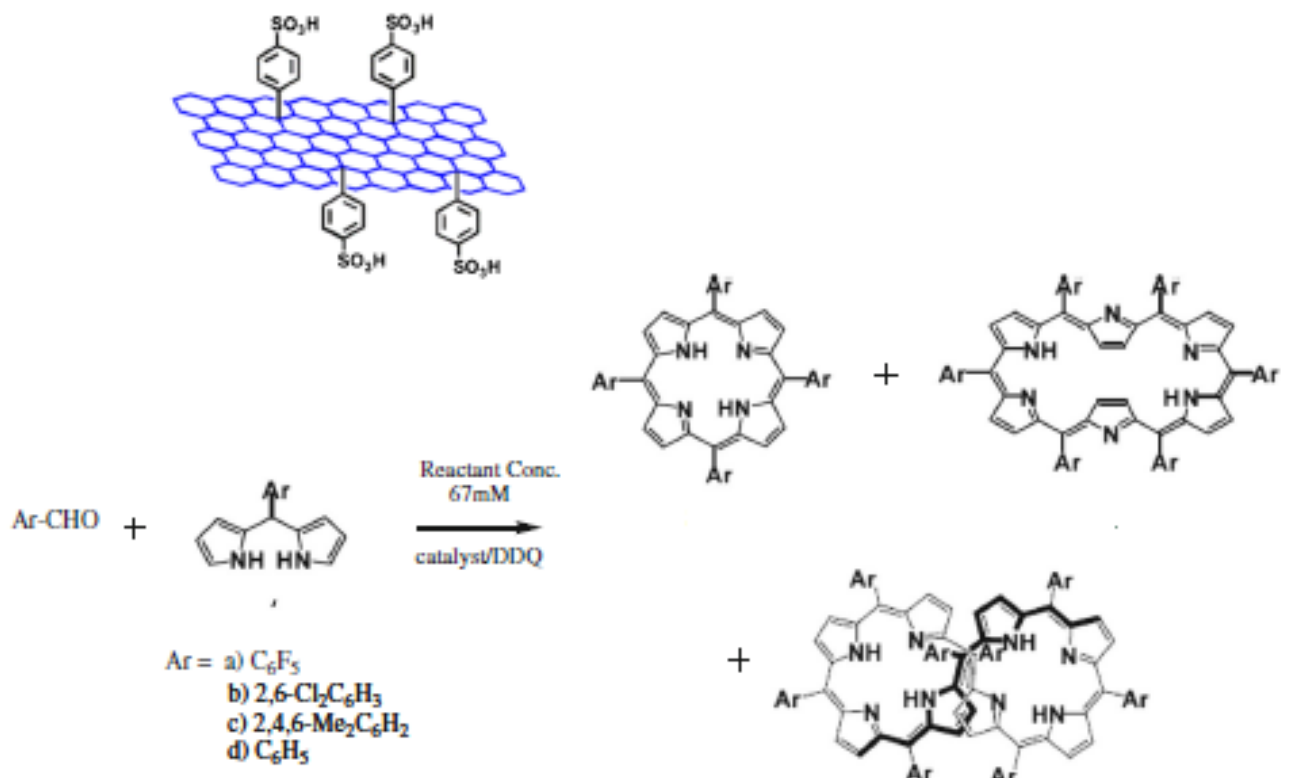

Figure 8. Synthesis of porphyrinic compounds in the presence of benzenesulfonic acid units covalently anchored on graphene starting from dipyrromethane and aromatic aldehydes. Adapted from ref. [35].

\section{Supramolecular architectures with carbon nanotubes}

Also in the case of CNTs, pyrene tags have been used as handles to attach catalytic active sites on these carbon materials through strong van der Waals $\pi-\pi$ interaction. One of these 
examples has been used to develop an enantioselective catalyst used in asymmetric syntheses. Thus, chiral diphosphine-modified CNT was prepared by adsorption of pyrene-modified $(R, R)$ rhodium (I) pyrphos complex onto carbon nanotubes (Scheme 2) via $\pi-\pi$ stacking. The rhodium complex remains strongly adsorbed on CNTs in various solvents allowing an easy recycling of the catalyst. This novel platform allows the performance of the asymmetric reaction under "pseudohomogeneous" conditions. Recycling of the chiral catalyst at the end of the reaction can be achieved via general solid/liquid separation simply by changing the solvent. The asymmetric hydrogenation of $\alpha$-dehydroamino esters occurred with enantiomeric excess higher than $92 \%$ [36].

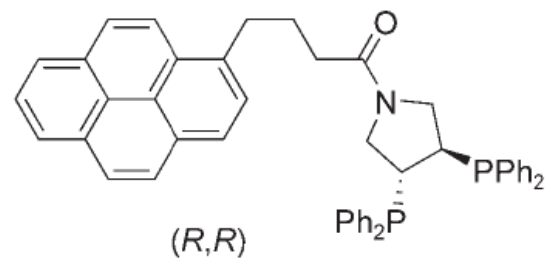

Scheme 2. Pyrenyl-modified pyrphos ligand able to attach to CNTs through the pyrenyl tag and form a complex with Rh (I) through the diphenylphosphine units.

Early in 1998, Martin et al. showed that pre-filled iron NPs into carbon nanotubes can catalyze under conventional chemical vapor deposition conditions the growth of inner tubes within the pristine external tube [37]. The driving force for the formation of this tube-in-tube multi-walled CNTs (MWCNTs) is the supramolecular association caused by strong $\pi-\pi$ interactions.

An even more versatile method to construct supramolecular well-assembled tube-in-tube structures is the one using graphitic carbon impurities as carbon source of the graphene sheets. 
These graphene tubes are the building-blocks for novel tube-in-tube nanostructures as shown in Figure 9 [38]. The formation of such MWCNTs is a reflection of the templation derived from the assembling process and the absence of covalent bonds among the pristine CNT and the growing small graphene segments. MWCNTs have found a wide range applications in catalysis, and gas storage and sensing.
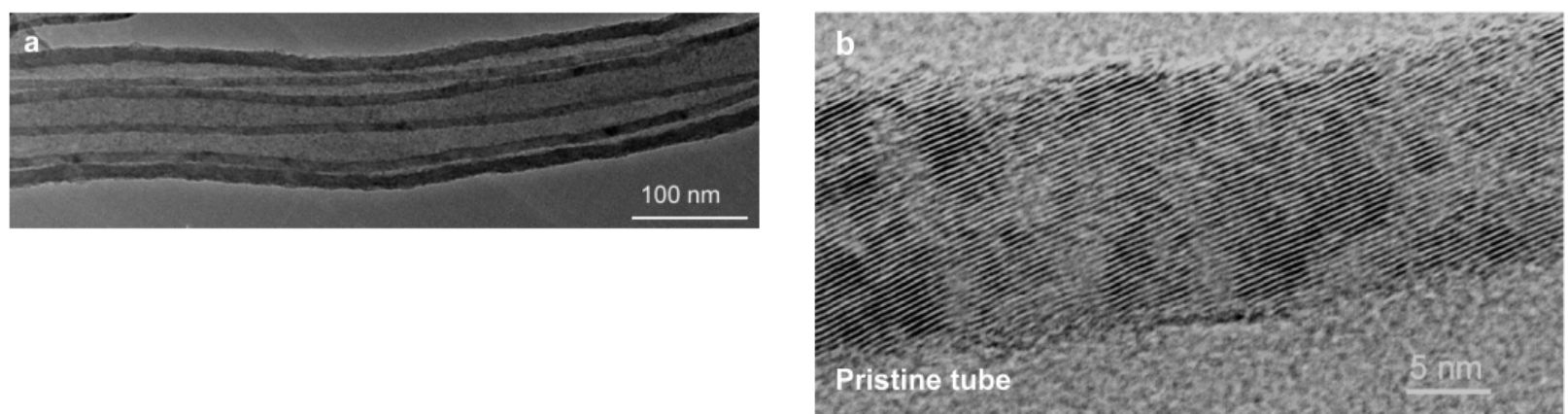

Figure 9. (a) TEM image of a triple-channel tube-in-tube MWCNT formed from simultaneous exterior and interior assembly of graphene sheets. (b) High-resolution TEM image of a representative pristine nanotube. With permission from ref. [38].

The use of CNTs as components for supramolecular architectures in combination of ionic liquids (ILs) was firstly reported by Fukushima and co-workers who demonstrated a molecular ordering of organic molten salts, such as 1-butyl-3-methylimidazolium $\left(\mathrm{BMIM}^{+}\right)$, triggered by single-walled carbon nanotubes (SWCNTs) [39]. Characterization of the phase transition and rheological properties of these gels ("bucky gels") have demonstrated that they possess a spatial arrangement due to the cross-linking of the nanotube bundles mediated by local molecular ordering 
of ILs (Figure 10). Such structures allowed the fabrication of highly electroconductive polymer/nanotube composites with a substantial enhancement in dynamic hardness.
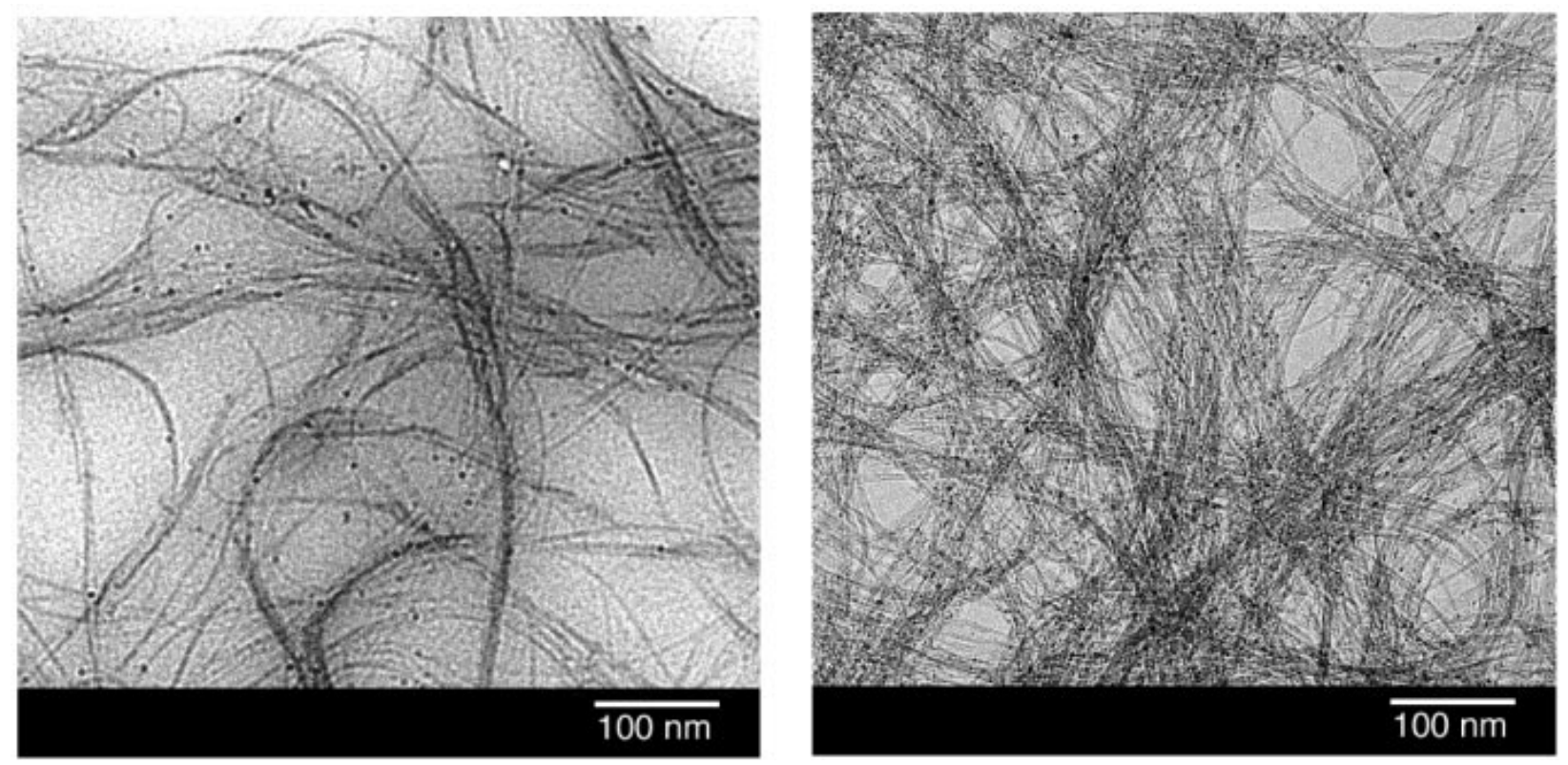

Figure 10. Morphologic changes produced by dispersing SWCNT (left) in $\mathrm{BMIMBF}_{4}$ forming bucky gel. Reproduced with permission from ref. [39].

Since this initial report, the interest for the production and use of the supramolecular noncovalent coated CNT-IL structures expanded. Several applications in different fields from electrochemistry to polymer composites have been reported [40]. These structures allow improving the performances of devices and composites in various areas.

As Raman and IR spectroscopy suggested, the supramolecular CNT-IL structures are generated through the $\pi-\pi$ interactions between IL cations and the nanotube walls [41]. Based on this, it is possible to develop novel multifunctional materials by combining the individual properties of both ILs and CNT, such as the high ionic conductivity and solvation ability of ILs. 
The non-covalent association and type of IL also influences the catalytic activity of the new materials.

Such supramolecular structures have been used as "hosts" for the deposition of metal NPs with the aim to be utilized for catalysis. For the Pt/MWCNT composites Chu et al. [42] performed control experiments demonstrating that the presence of ILs preserves the size and dispersion of Pt NPs Figure 11. Furthermore, this strategy makes possible the synthesis of Pt NPs with tunable sizes, allowing the study of their size-dependent catalytic activity and selectivity in various reactions. As an example, glycerol oxidation may lead to the formation of glyceraldehyde (GLYA), glyceric acid (HYPAC) or glycolic acid (GLYCA) (Scheme 3). The TOF value and the selectivity in this reaction to these products is clearly influenced by the Pt NP size. Chun et al utilized a quite similar approach to immobilize Pd NPs and investigated the resulting catalysts for the hydrogenation of different olefins [43].

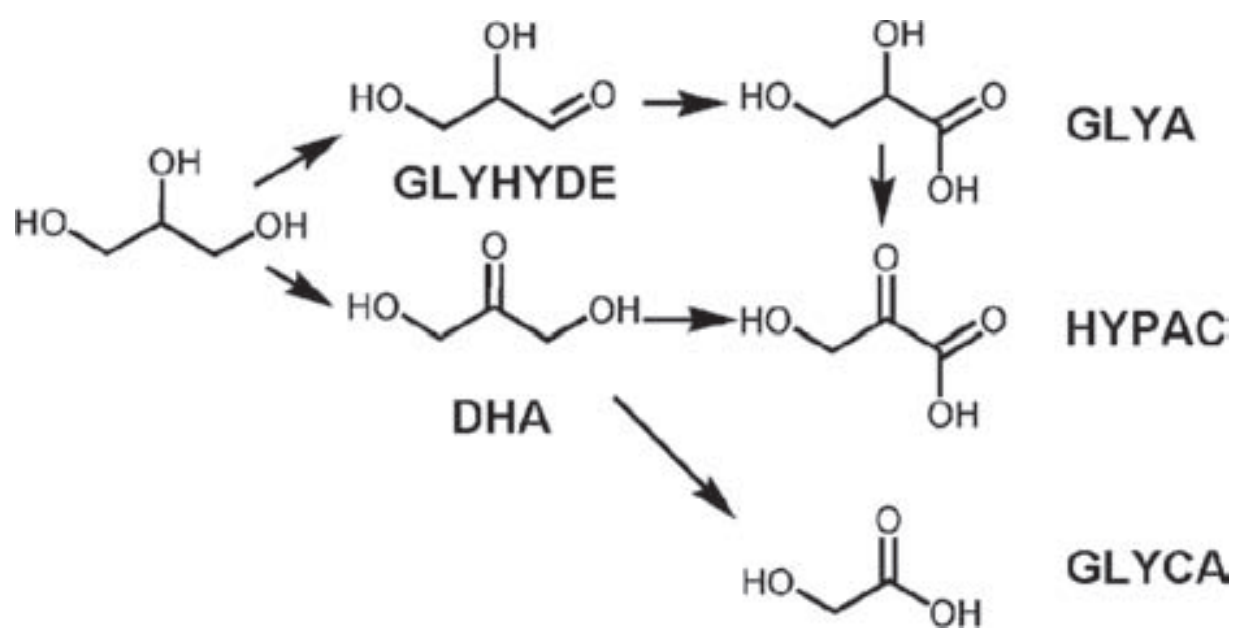

Scheme 3. Products of glycerol oxidation catalyzed by Pt NPs on IL-MWCNT. 

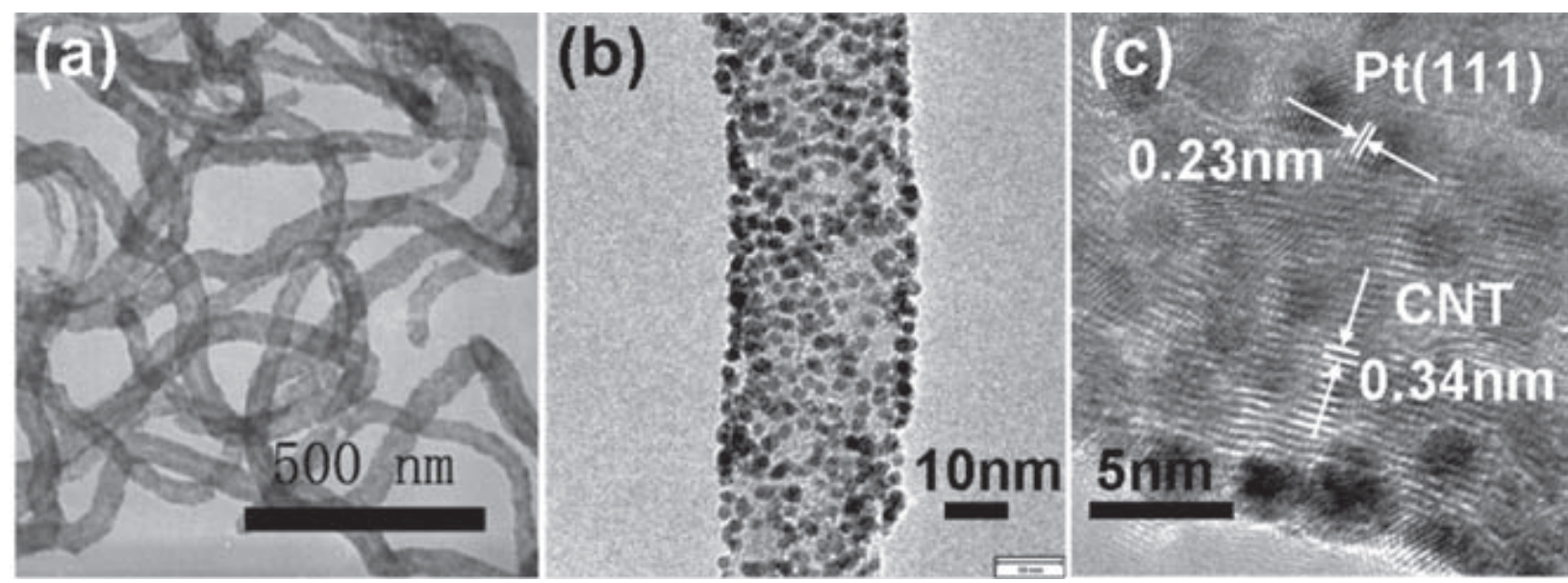

Figure 11. TEM (a) and HRTEM (b,c) images of the Pt/[BMIM]BF $4 / M W C N T$ (20 wt\% Pt) composites. Reproduced with permission from ref. [42].

A very close approach has also been considered by Gruttadauria and co-workers to obtain a $\mathrm{PdCl}_{4} \mathrm{IL} / \mathrm{MWCNT}$ catalyst to promote the Suzuki-Miyaura carbon-carbon cross-coupling reaction [44]. Preparation of these catalysts was performed attaching non-covalently any of the two bis-imidazolium dibromide derivatives having phenyl or pyrenyl groups to the walls of MWCNT (Figure 12). The aromatic tags allow anchoring of ILs, while the presence of the two imidazolium cations helped the immobilisation of $\mathrm{PdCl}_{4}{ }^{-}$ions by exchange with the bromide ions. The resulting catalyst was stable enough to be recycled with only a minor loss in activity. HRTEM images clearly showed the presence of some material on the external walls of MWCNTs that was attributed to the existence of bis(pyren-1-ylmethylimidazolium) aggregates. 

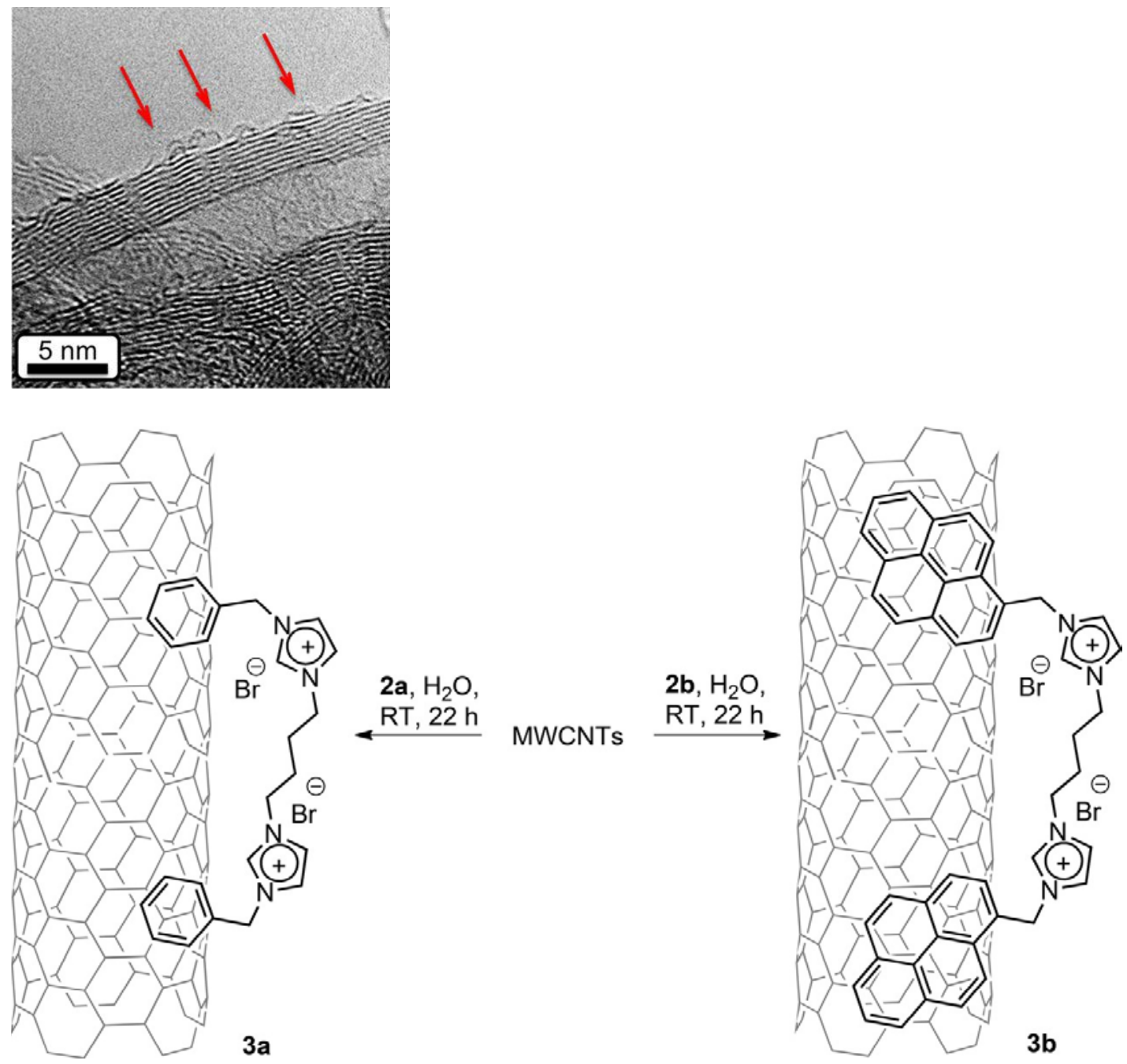

Figure 12. Top: High resolution TEM images of $\mathrm{PdCl}_{4} \mathrm{IL} / \mathrm{MWCNT}$. Bottom: Non-covalent immobilization of ILs onto MWCNTs. a) schematic structure of the supramolecular aggregates. With permission from ref. [44].

The use of the so-called IL-assisted sonochemical method, in combination with the supramolecular chemistry between ILs and CNTs, affords a more facile control in the size, amount and interfacial contact of uniformly decorated NPs (Figure 13) [45]. Accordingly this procedure allows to control the size of various NPs including Pt, Pd, Au, Ag, SnOx, FeOx, ZnOx. Their 
catalytic performance confirmed the advantages of the preparation protocol. Thus, the Pt/BMIMBF $4 / \mathrm{MWCNT}$ supramolecular ensemble has better electrochemical performance than pristine MWCNTs and Pt/MWCNT for both, oxygen reduction and methanol oxidation.

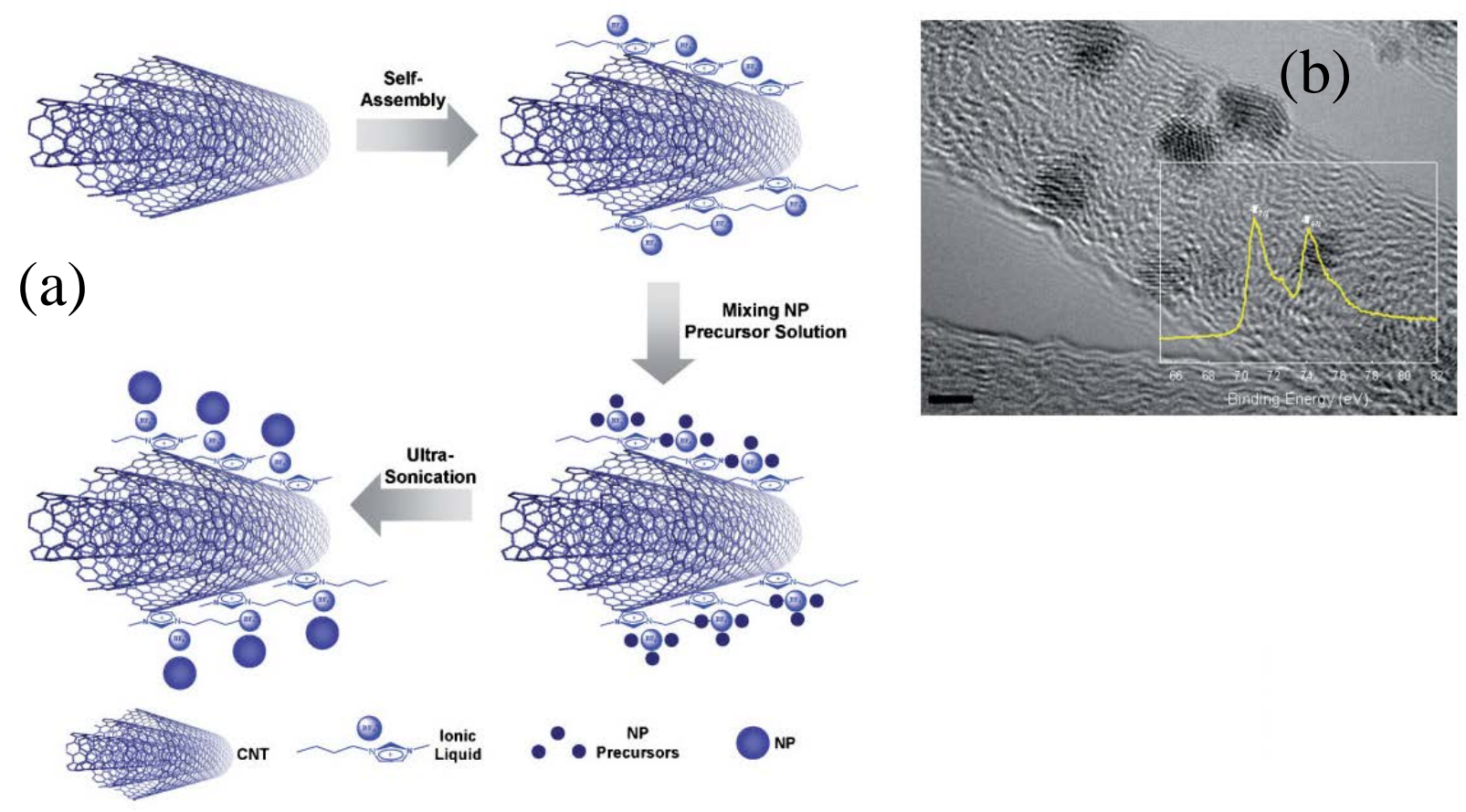

Figure 13. Illustration of the `preparation procedure (a), TEM image and XPS Pt 4f peak (b) of Pt/IL/MWCNT. Reproduced with permission from ref. [45].

Metal NPs supported onto a porous support can be obtained by deposition of stabilized colloidal suspensions [46]. However, under reaction conditions the stability of these NPs is not generally high and they can suffer agglomeration resulting in a negative effect onto the catalytic performance. In this context, it has been reported that cyclodextrins as metal NP protective agents may eliminate this disadvantage. Accordingly, the preparation strategy considers adsorption onto the carbon support the metal NPs already stabilized by cyclodextrins [46-49]. These supramolecular catalysts exhibit for the hydrogenation of xylene isomers a very good thermal 
stability and provide higher TOF values and better selectivities than metal NPs directly supported on carbon.

Photocatalytic aerobic oxidation of amines to form imines has been reported using a modified carbon nitride having carbonyl groups on its surface as a metal-free photocatalyst. The material was prepared via the supramolecular assembly of cyanamide induced by ammonium sulfate. The resulting $\mathrm{C}=\mathrm{O}$-modified mesoporous carbon nitride is able to bind $\mathrm{H}_{2} \mathrm{O}_{2}$ formed from $\mathrm{O}_{2}$ and to accelerate the reaction between activated $\mathrm{H}_{2} \mathrm{O}_{2}$ and amines under ambient conditions [50].

Nitrogen-functionalized CNTs [(N)CNTs] have also been considered for the production of supramolecular assembles. Figure 14 depicts an example of these structures, where the functional group is composed of a heteropoly acid (HPA) [51]. The interaction between HPA and (N)CNT is mainly electrostatic attraction, due to the presence of opposite positive [(N)CNT] and negative $\left(\mathrm{PA}^{-}\right)$charges. This Coulombic attraction also ensures a relatively high stability and easy regeneration of the catalysts. These materials have been evaluated as catalysts for ester hydrolysis reactions, a typical reaction promoted by HPA.

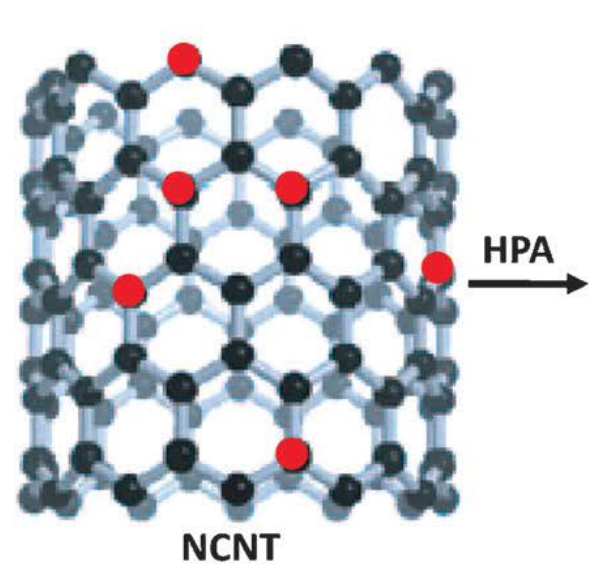

NCNT

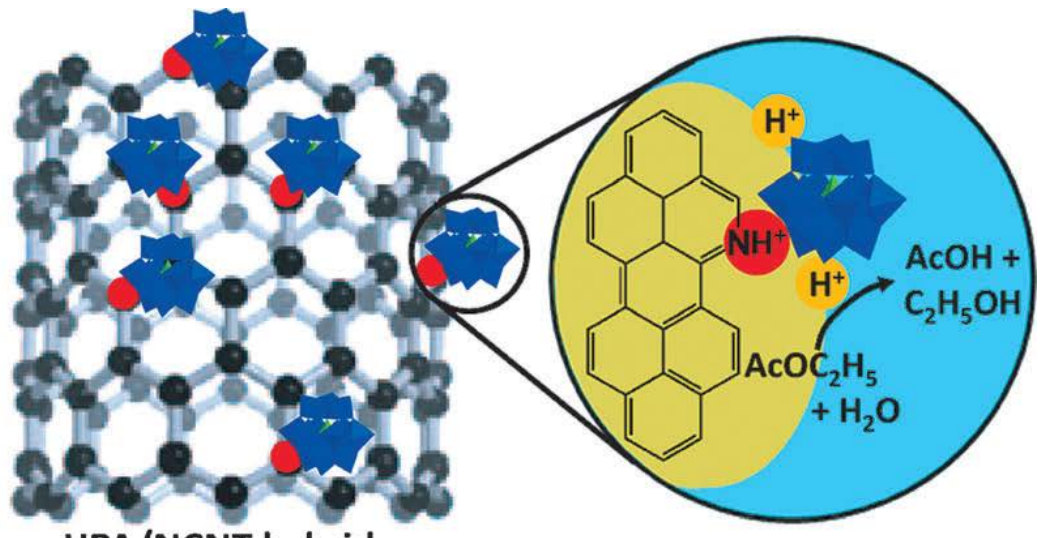

HPA/NCNT hybrid 
Figure 14. Preparation of the $\mathrm{PA}^{-} /\left(\mathrm{H}^{+} \mathrm{N}\right) \mathrm{CNT}$ hybrid by acid-base neutralization and illustration of its catalytic activity to promote ester hydrolysis. Reprinted with permission from ref. [51].

\section{Supramolecular structures in biocatalysis}

Supramolecular structures based on CNTs have also been considered in biocatalysis. A very original example has connected electrically redox proteins and metallic electrodes using tipmodified CNTs. These tip-modified CNTs establish non-covalent interactions with the metal surface through thiol groups [52]. Figure 15 illustrates the way in which the enzyme was attached to the electrode. 


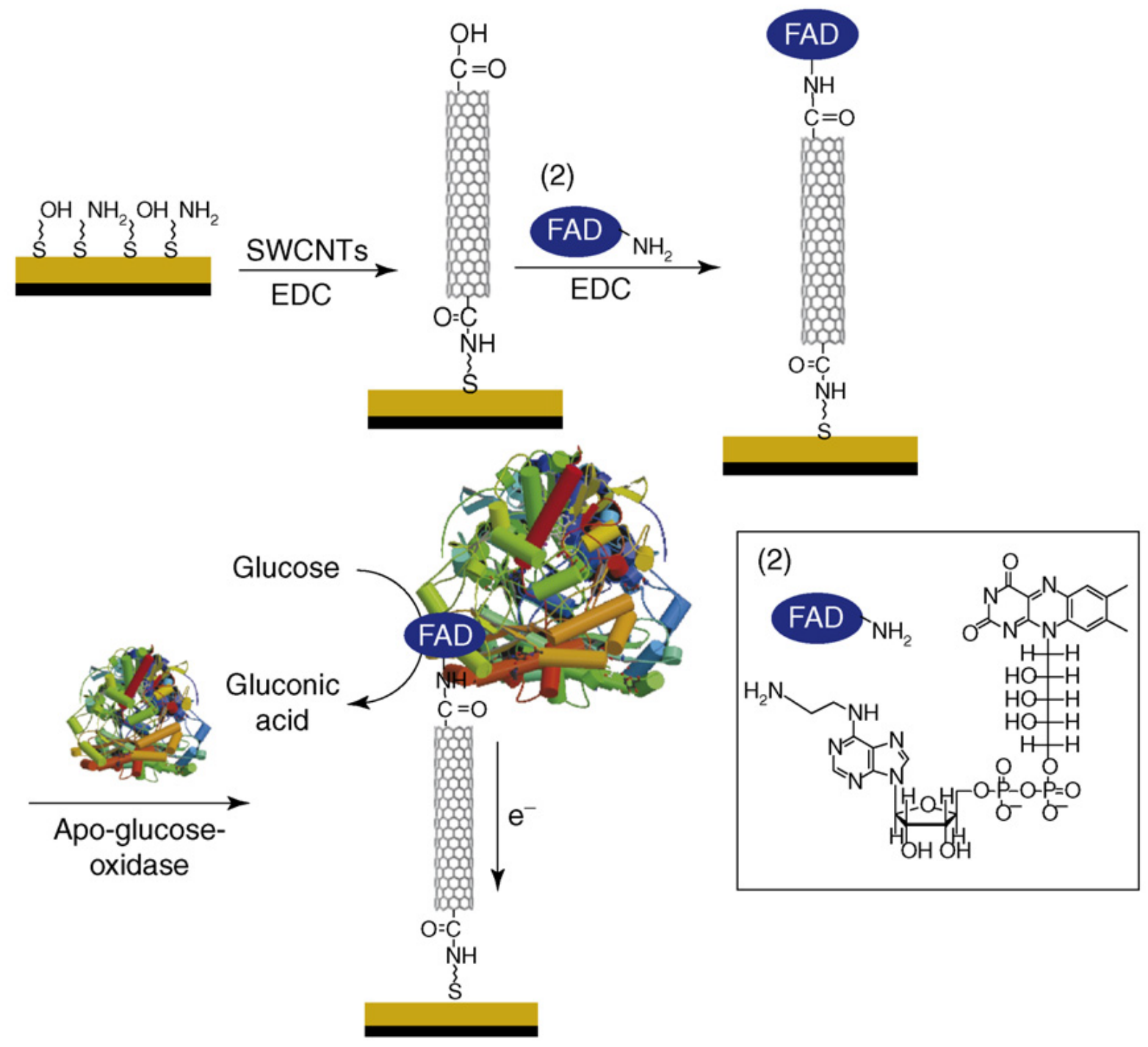

Figure 15. Steps for connecting electrically glucose oxidase to a gold electrode using CNTs as connectors. The process is based on the reconstitution of apo-glucose oxidase (apo-GOx) with a FAD cofactor that is attached to SWCNT. SWCNT is associated with a gold electrode by Au-S interaction. EDC refers to 1-ethyl-3-[3-dimethylaminopropyl]carbodiimide Reproduced with permission from ref. [52].

In another example by the same group, an electron-poor bis bipyridinium cyclophane was threaded on a molecular wire containing electron-rich bis iminobenzene units assembled on the 
metallic electrode. The interaction between the mobile positive bipyridinium units and the bis iminobenzene groups was via formation of a charge-transfer supramolecular complex. In this charge-transfer complex. the bipyridium cation acts as acceptor and the bis iminobenzene unit in the molecular wire as electron donor. The wire was then 'stoppered' either by an adamantane unit or by a flavin adenine dinucleotide (FAD) (Figure 16). In this way, the bis bipyridinium cyclophane is free to move along the wire finding the most suitable station to establish the stronger interaction. However, it cannot become dethreaded from the wire due to the large size of the bulky stopper. The cyclophane unit can, then, change the position in the wire between two stations depending on the electrochemical input. Thus, in the oxidized state the cyclophane unit migrates from the electrode to the $\pi$-donor bis iminobenzene unit to form the charge-transfer complex. This complex is destroyed and the migration reversed to the initial station of the cyclophane on the molecular wire by chemical or electrochemical reduction of the bipyridinium shuttle. When the stopper is FAD, this cofactor can associate with glucose oxidase. Operation of the enzymatic reaction causes the bipyridinium cyclophane shuttle to become reduced moving towards the electrode. At this point, the bipyridinium cyclophane becomes oxidized and returns to the bis imino station. In this way, the cyclophane is transporting electrons from the enzyme to the metal electrode and operation of the enzymatic reaction can be established by monitoring the movement of the bipyridinium shuttle.[52] 
(a)

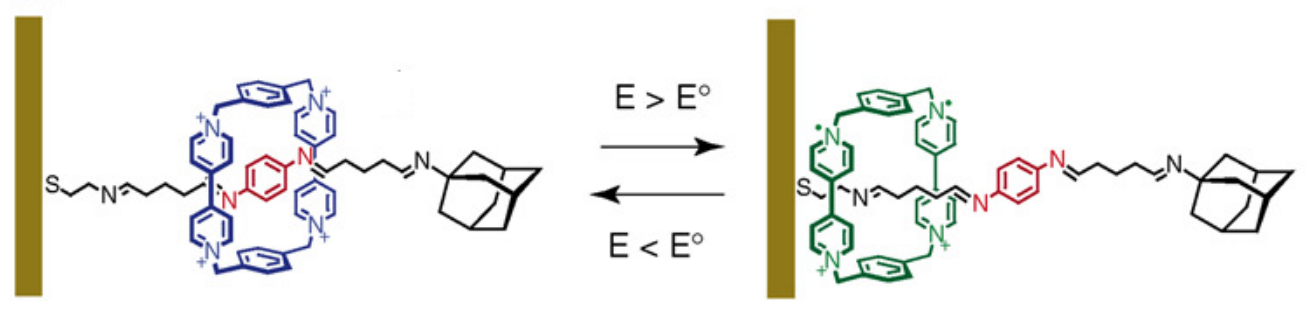

(b)
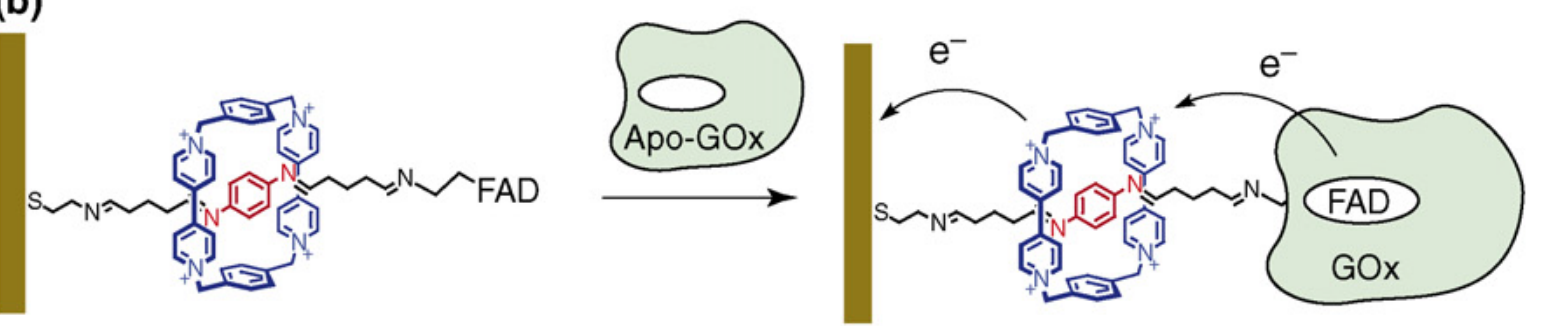

Figure 16. (a) Electrochemically induced reversible translocation of cyclophane unit along an adamantly bis imino molecular wire. (b) The reconstitution of apo-glucose oxidase (apo-GOx) with a FAD cofactor that is the 'stopper' of the cyclophane shuttle on the molecular wire. The redox enzyme is contacted with the electrode by the bis pyridinium cyclophane shuttle transporting electrons along the wire. Reproduced with permission from ref. [52].

\section{Supramolecular approaches for the synthesis of structured materials}

Cycloparaphenylenes (CPPs) can be considered as the smallest segment of armchair CNTs. The use of CPPs as substrates for the bottom-up preparation of CNTs has been proposed as a potential synthesis of structurally uniform CNTs, with precisely controlled chirality. This hypothesis has been already confirmed by the contributions of several groups [53-57].

In one of these examples, CPPs are produced starting from diborylated tetramesityl that is complexed by $\mathrm{Pt}(\mathrm{COD}) \mathrm{Cl}_{2}$ (COD: 1,4-cyclooctadiene) in the presence of CsF in tetrahydrofuran, leading to a Pt-bridged macrocyclic intermediate (Figure 17). CPPs have a favorable structure to 
establish supramolecular association affording, for example, the selective complexation with fullerenes via van der Waals interaction. Such a behavior has been demonstrated by fluorescence quenching experiments and molecular dynamic simulations [57].
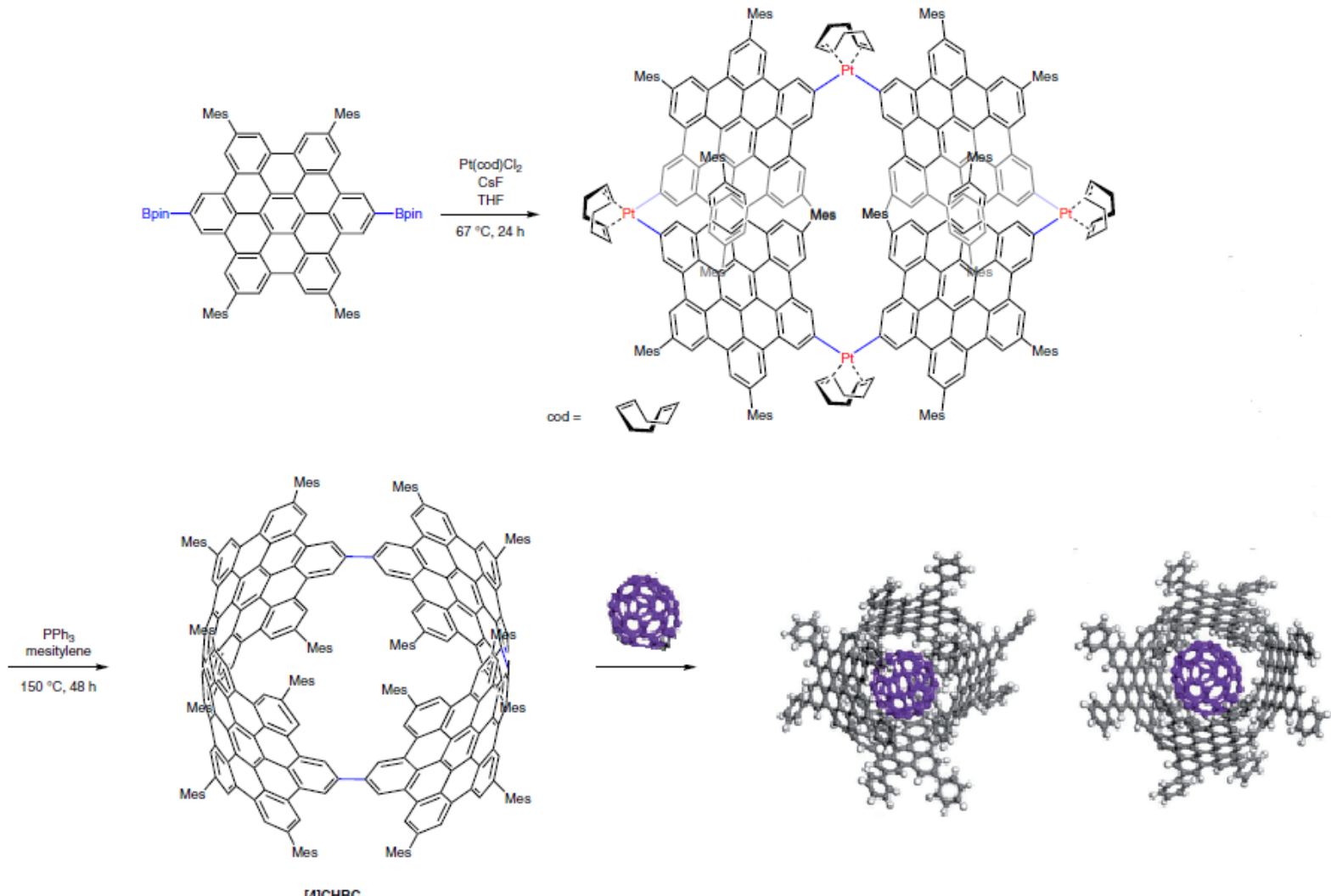

Figure 17. Selective complexation of fullerenes by CPPs via supramolecular concave-convex $\pi-\pi$ interactions. Reproduced with permission from ref. [57].

Tunable and robust 2D molecular arrays with desirable lattices and functionalities over a macroscopic length scale have also been prepared based on halogen bonding [58]. This was possible using the $\mathrm{Au}(111)$ surface which is able to catalyze the dehalogenation of hexabromobenzene (HBB). Debromination affords negatively charged bromide adatoms $\left(\mathrm{Br}^{\delta^{-}}\right)$ able to generate the targeted assemblies via a $\mathrm{C}-\mathrm{Br}^{\delta+} \ldots \mathrm{Br}^{\delta^{-}}$interaction. These superstructures 
(including densely packed hexagonal, tetragonal, dimer chain, and expanded hexagonal lattices) can be produced even at room temperature. The role $\mathrm{Br}^{\delta^{-}}$regulating lattice characteristics, having impact in molecular nanoelectronics and 2D crystal engineering, is critical. Figure 18 depicts the formation of the $\mathrm{Br}^{\delta-}$ adatom and the phase transition to the tetragonal phase.
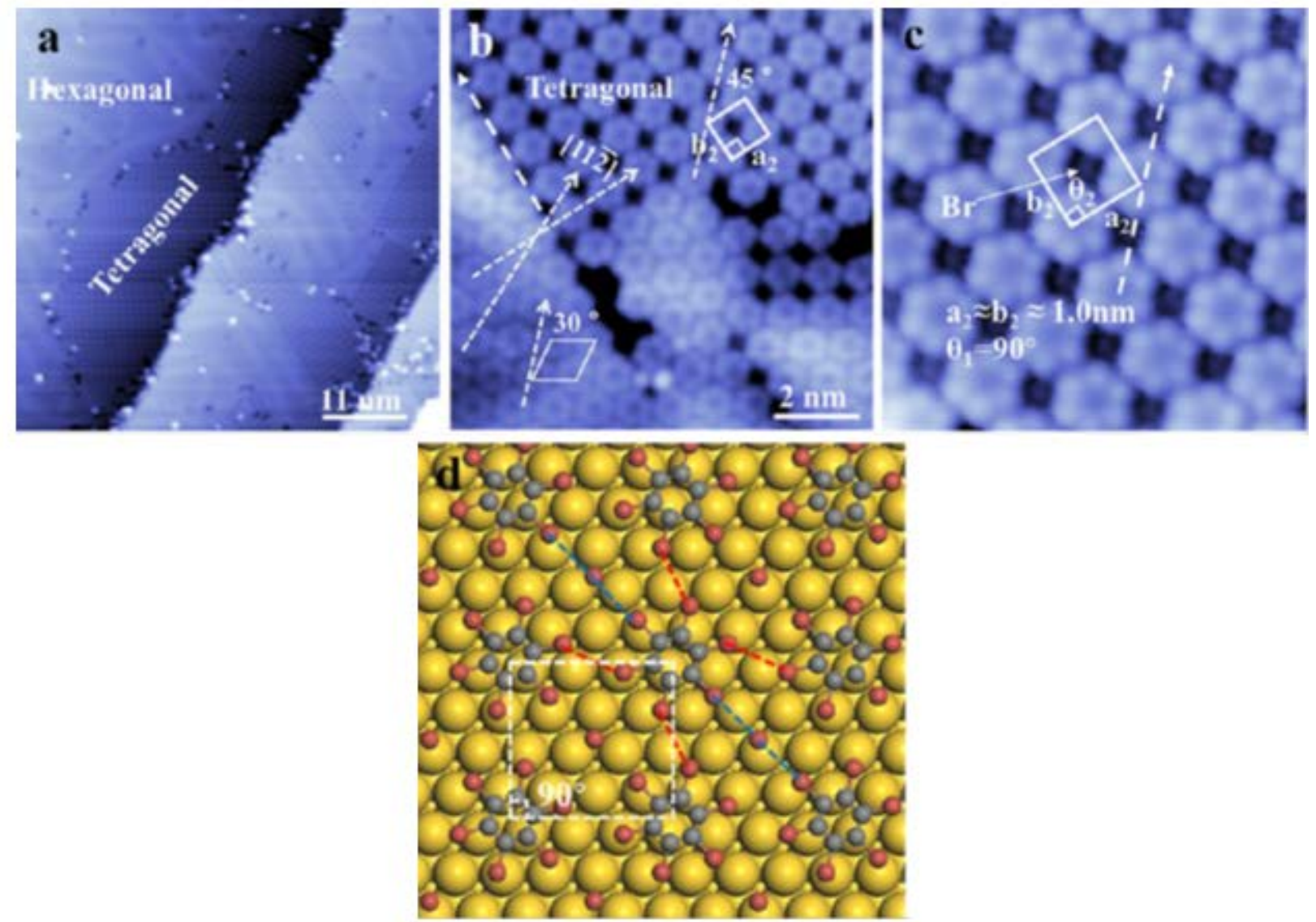

Figure 18. $\mathrm{Br}^{\delta-}$ adatom formation and phase transition to tetragonal phase. (a) Carbon clusters formed by dissociation of HBB molecules and tetragonal phase of HBB on Au (111) after annealing at $60{ }^{\circ} \mathrm{C}(1 \mathrm{~V}, 200 \mathrm{pA})$. (b) Enlarged view of a domain boundary between hexagonal and tetragonal patterns (200 mV, $500 \mathrm{pA})$. (c) Molecularly resolved STM image showing the presence of an adatom at the center of each unit cell (-200 mV, $500 \mathrm{pA})$. (d) Top side view of the 
DFT optimized structure. White dashed lines indicate the unit cell. Halogen bonding between HBB molecules, $\mathrm{HBB}$ and $\mathrm{Br}^{\delta-}$ adatoms are marked by red and blue dashed lines, respectively. Adsorption distances of $\mathrm{HBB}$ and $\mathrm{Br}$ adatoms are indicated in the panels. Reprinted with permission from ref. [58].

Organic capsules with accessible empty interior as cucurbit[n]urils (Q[n]s) may also be building blocks for the production of nanoporous assemblies. They present a rigid hydrophobic cavity flanked by " $\mathrm{n}$ " polar $\mathrm{C}=\mathrm{O}$ groups at the portals that are able to establish strong charge-dipole and hydrogen bonding interactions. These organic capsules are able to form strong association complexes with organic molecules and inorganic ions (Figure 19) [59]. Other weak noncovalent interactions such as hydrogen bonding and $\pi \cdots \pi$ stacking, as well as $\mathrm{C}-\mathrm{H} \cdots \pi$ and ion-dipole interactions, could help as "outer-surface interactions”. These weak forces can lead to the formation of even more elaborated structures involving the presence of metals or interaction with aromatic entities. Figure 20 presents examples of such structures. Due to the presence of metals, the resulting supramolecular entities may exhibit multiple catalytic behavior in either hydrogenation or oxidation reactions. 


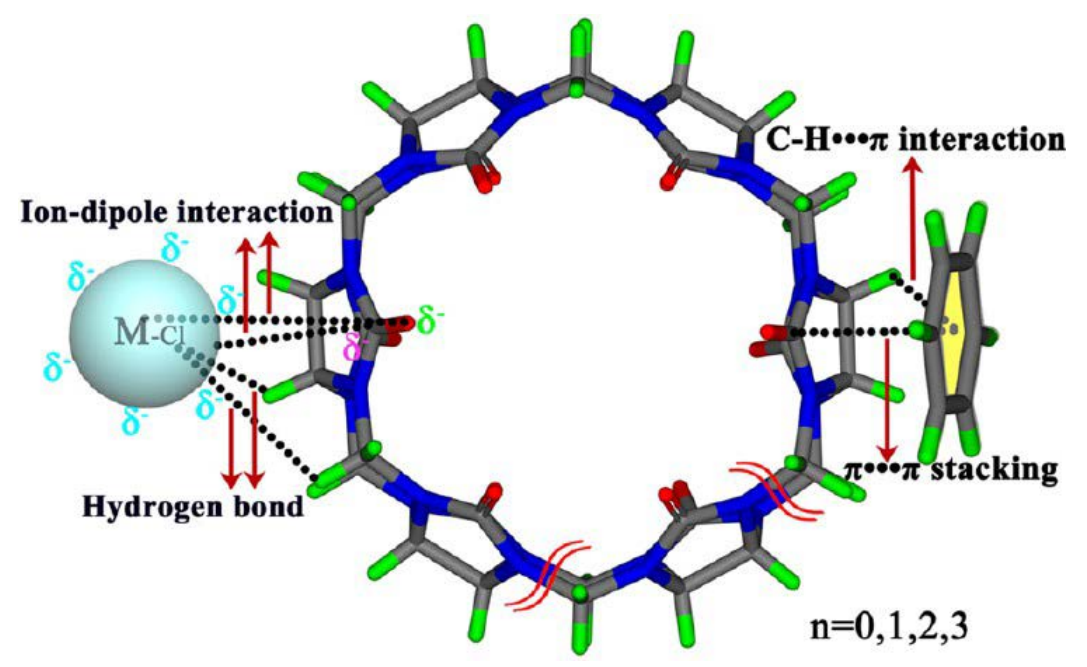

Figure 19. Q[n] structures and potential interactions that can be established with guests. Taken with permission from ref. [59].

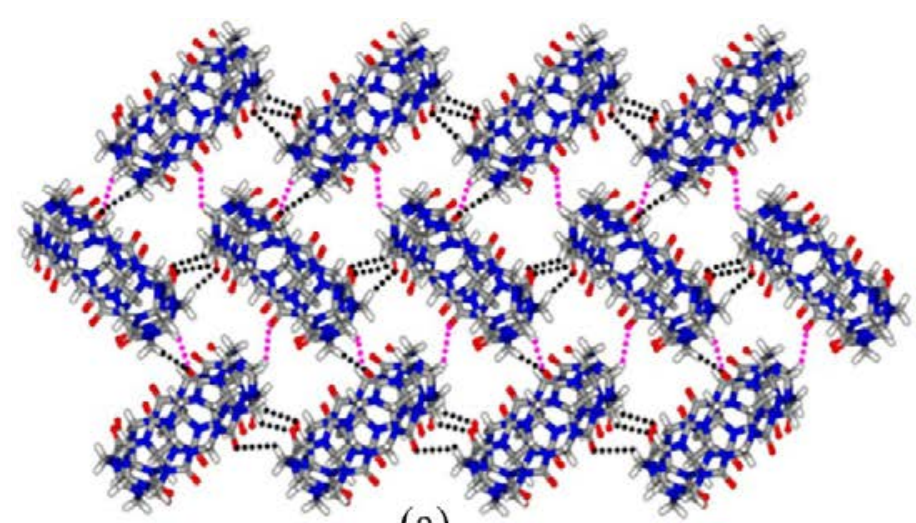

(a)

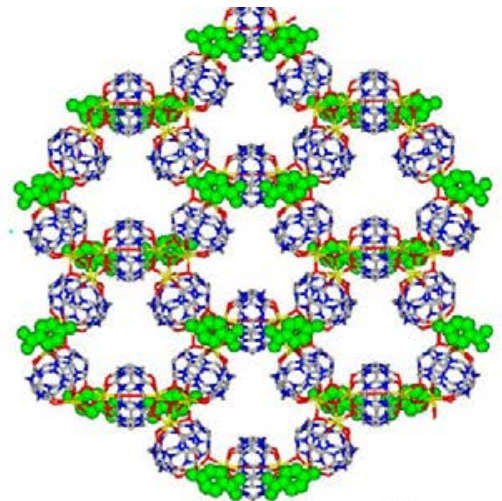

(b)

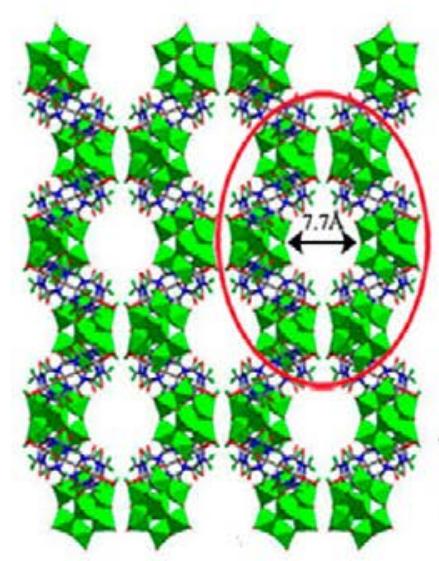

(c)

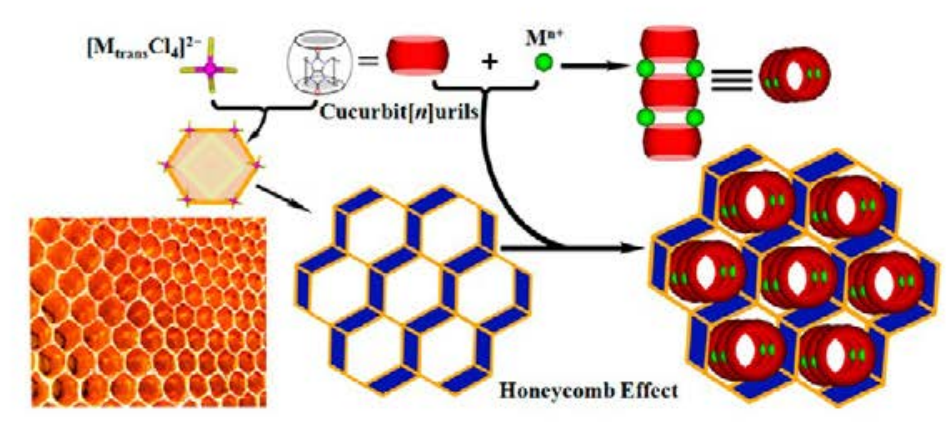

(d)

Figure 20. Examples of supramolecular structures based on supramolecular assembly of Q[n]: 
(a) the fiber-like Q[7]-based supramolecular entities; (b) six-membered Q[5] rings with molecules of p-hydroxybenzoic acid (green) in a single layer; (c) $(\mathrm{HO})_{10} \mathrm{Q}[5] /\left[\mathrm{PMo}_{12} \mathrm{O}_{40}\right]_{3}$ supramolecular assembly; (d) honeycomb structures derived from Q[n]-based coordination. Reprinted with permission from ref. [60].

In another approach Q[8]-based porous materials were produced in aqueous solution by recrystallization with a suitable molecular chaperone [61]. Figure 21 depicts according to X-ray crystallography some of the supramolecular structures formed in which the inner diameter was around $0.6 \mathrm{~nm}$. These materials exhibit selective adsorption properties of $\mathrm{CO}_{2}$ over $\mathrm{CH}_{4}, \mathrm{~N}_{2}$ and $\mathrm{H}_{2}$.

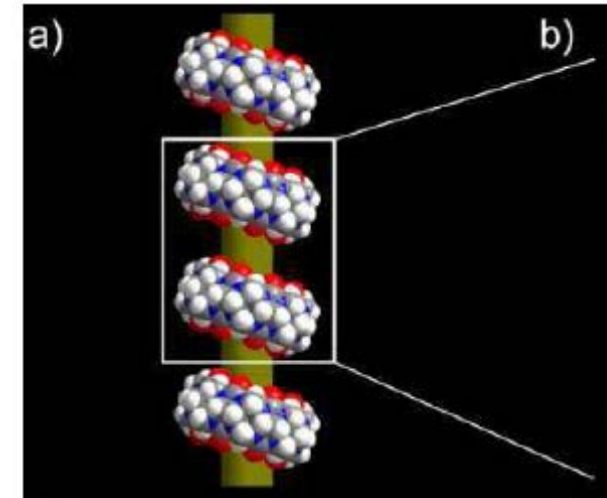

d)

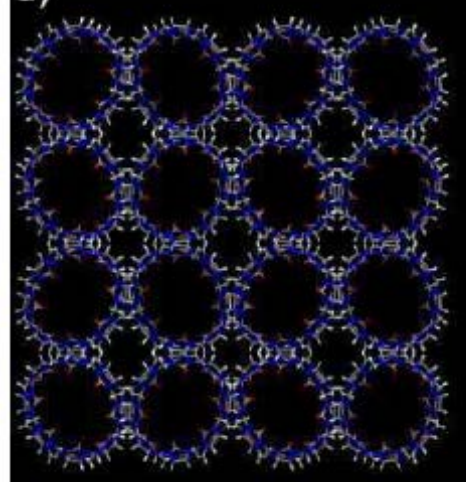

dich dis dis Hith the thit or WT Mrot ir

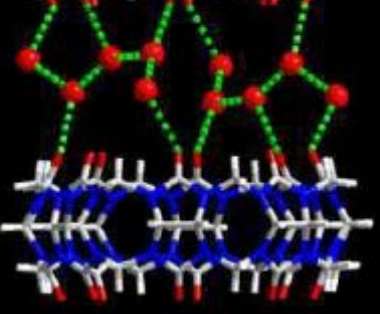

e)

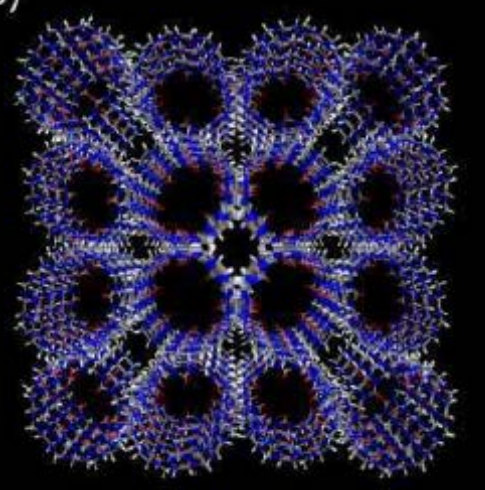

c)

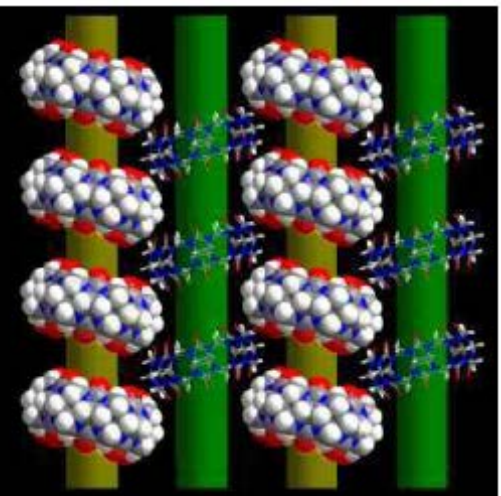

f)

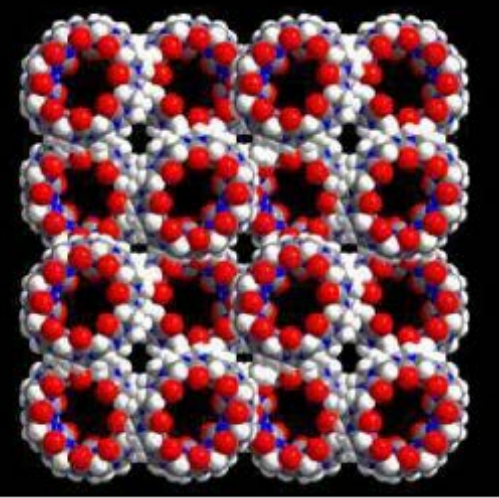


Figure 21. Structure determined by X-ray crystallography of the supramolecular assemblies formed by Q[8]: a) side view of 1D nanotube; b) two water chains between two Q[8] molecules in 1D nanotube; c) side view of the nanotubular 1D channels; d) top view; e) perspective view and f) space-filling view of the nanotubular framework from c axis. Color code: N-blue, O-red, C-gray. H-bonds-green dotted line. Water molecules not involved in hydrogen bonding and bromide ions are omitted for charity. Reproduced with permission from ref. [61].

Perylenetetracarboxylic diimide (PDI) derivatives modified with linear alkylcarboxysubstituent side-chains of different lenghts (Figure 22) can also aggregate to construct H/J-type supramolecular nanostructures (Figure 1). Excited state PDI $\mathrm{H}$-aggregates mainly forms $\cdot \mathrm{O}_{2}{ }^{-}$and $\mathrm{h}^{+}$by electron-transfer (ET), whereas, excited state PDI J-aggregates mainly generates ${ }^{1} \mathrm{O}_{2}$ via energy-transfer (EnT). As an effect of the stronger oxidizability of $\cdot \mathrm{O}_{2}{ }^{-}$and $\mathrm{h}^{+}$, the $\mathrm{H}$ aggregates are able to promote water photooxidation under visible light, whereas, J-aggregates exhibit potential application in photodynamic therapy for skin cancer treatment owing to its high ${ }^{1} \mathrm{O}_{2}$ quantum yields under red light irradiation [6]. 


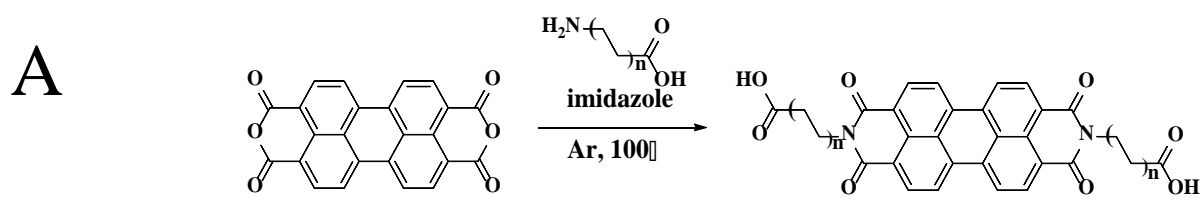

B

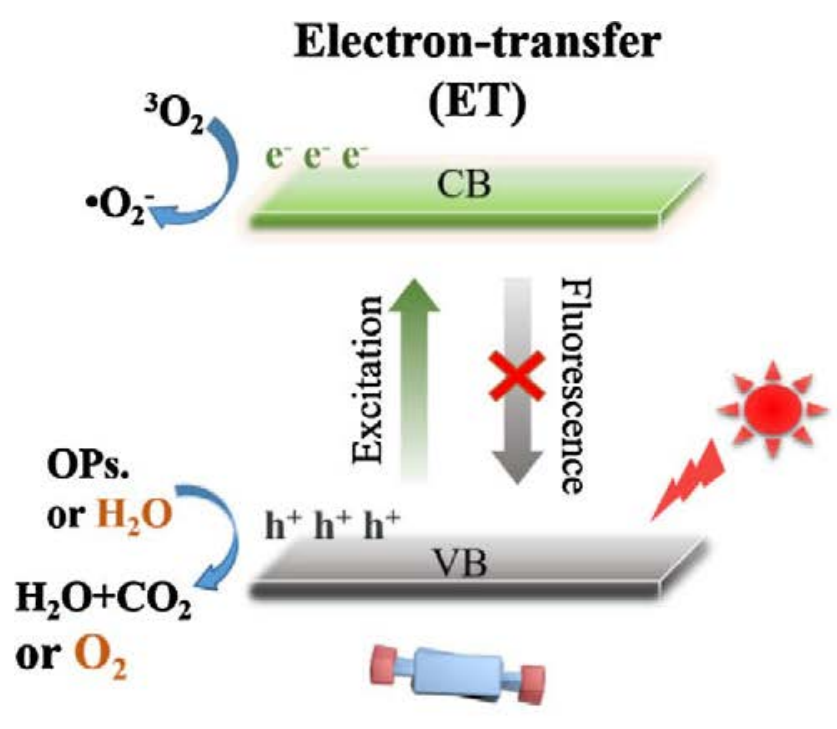

H-aggregates
Energy-transfer

(EnT)

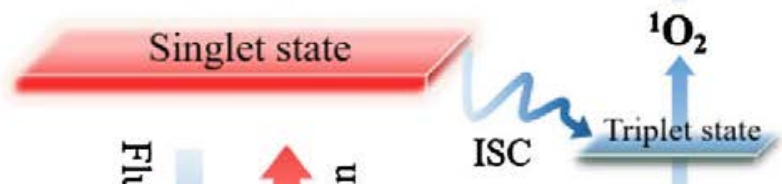

${ }^{3} \mathrm{O}_{2}$

Figure 22. Schematic illustration of general synthetic procedure for the preparation of H-PDI and J-PDI molecules (A) and the photocatalytic behavior (B) Adapted from ref. [6].

Supramolecular aggregates in Metal-organic frameworks (MOF) and Metal-organic channel (MOC) confined nanospaces

MOFs are metal-ligand coordination polymers with crystalline structure and large porosity [62-64]. The structure of these materials is also not based on covalent bonds, but on Coulombic attractions and coordinative bonds. Although a detailed description of MOFs is outside the scope of the review, these materials can also have supramolecular aggregates with application in catalysis including oxidation, C-C bond forming reactions, formation of heterocycles and in various media such as water or under solvent free conditions [64-67]. Thus, MOFs can also provide 
interpenetrated supramolecular structures with catalytic behavior. MOC structures represent another example of molecular self-assembly of metal ions as nodal units with designed ligands as spacers [68]. The use of MOFs and MOCs in supramolecular catalysis has been recently reviewed [69].

In one of the examples using MOFs to develop supramolecular catalysts, two isomeric supramolecular complexes of $\quad\left(\left[\mathrm{Cd}\left(\mathrm{NH}_{2}\right.\right.\right.$-bdc $\left.\left.)(\mathrm{bphz}) 0.5\right] \cdot \mathrm{DMF} \cdot \mathrm{H}_{2} \mathrm{O}\right\} \mathrm{n} \quad\left(\mathrm{NH}_{2}\right.$-bdc=2aminobenzenedicarboxylic acid, bphz=1,2-bis(4-pyridylmethylene)hydrazine) composed of two different ligands exhibit different structure and properties (Figure 23) [68]. The first structure has a secondary building unit (SBU) constituted by a $\mathrm{Cd}_{2}(\mathrm{COO})_{4}$ paddle wheel nodes. This isomeric MOF is flexible in nature. In contrast, the second MOF solid with the same formula has a rigid framework based on a $\mu$-oxobridged $\mathrm{Cd}_{2}(\mu-\mathrm{OCO})_{2}$ SBU. The presence of the $-\mathrm{NH}_{2}$ group was found to enable the $\mathrm{CO}_{2}$ adsorption, while in the case of the oxo-bridged isomer the basic $-\mathrm{NH}_{2}$ sites were further exploited as active sites to promote efficiently the Knoevenagel condensation [70]. 


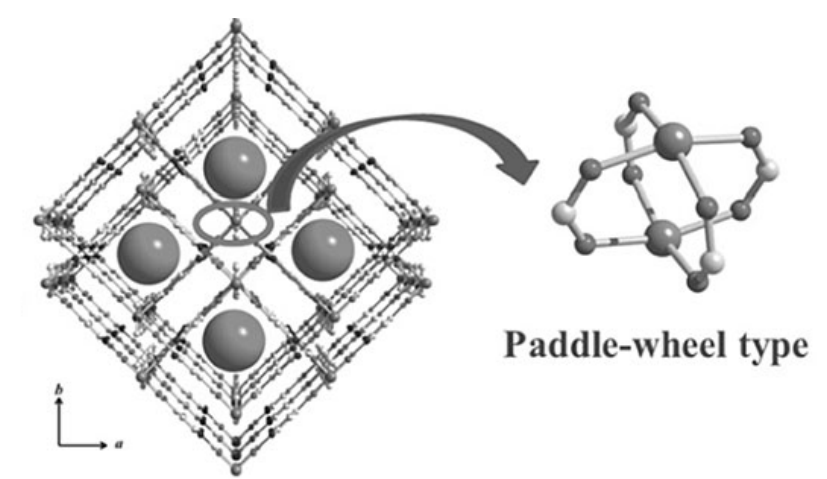

$\left.\left[\mathrm{Cd}\left(\mathrm{NH}_{2}-\mathrm{bdc}\right)(\mathrm{bphz})_{0.5} \mathrm{I} \cdot \mathrm{DMF} \cdot \mathrm{H}_{2} \mathrm{O}\right)\right\}_{n}(1)$

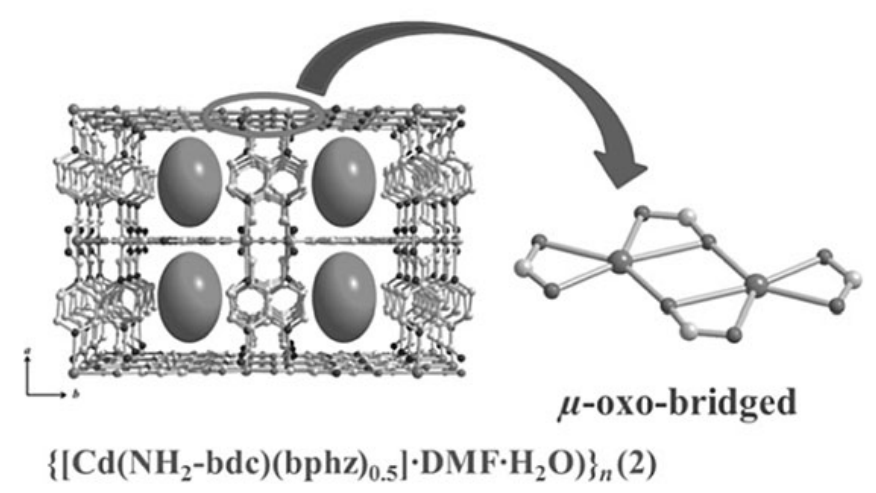

Figure 23. Structure of two supramolecular MOFs having the same composition. Reproduced with permission from ref. [70].

The design of multi-stimuli capped MOFs and supramolecular entities like [2] pseudorotaxanes can serve to develop a material with controllable properties. Thus, for a given concentration of $\mathrm{Ca}^{2+}$, the concentration of the encapsulated substrate and its release in a Zr-MOF can be controlled with the $\mathrm{pH}$ [71]. Such a design opens the possibility of developing smart biomaterials.

Highly stable zirconium/hafnium-based MOFs incorporating active species have been reported. These host-guest materials give rise to catalysts exhibiting higher performances or unusual activity compared with their homogeneous analogues. Thus, besides the catalytic functions incorporated at the zirconium (Hf)-oxide node or the linker, the guests encapsulated in 
the pores afford high selectivity in the hydrogenation of cinnamic aldehyde to cinnamic alcohol (Figure 24) [72].

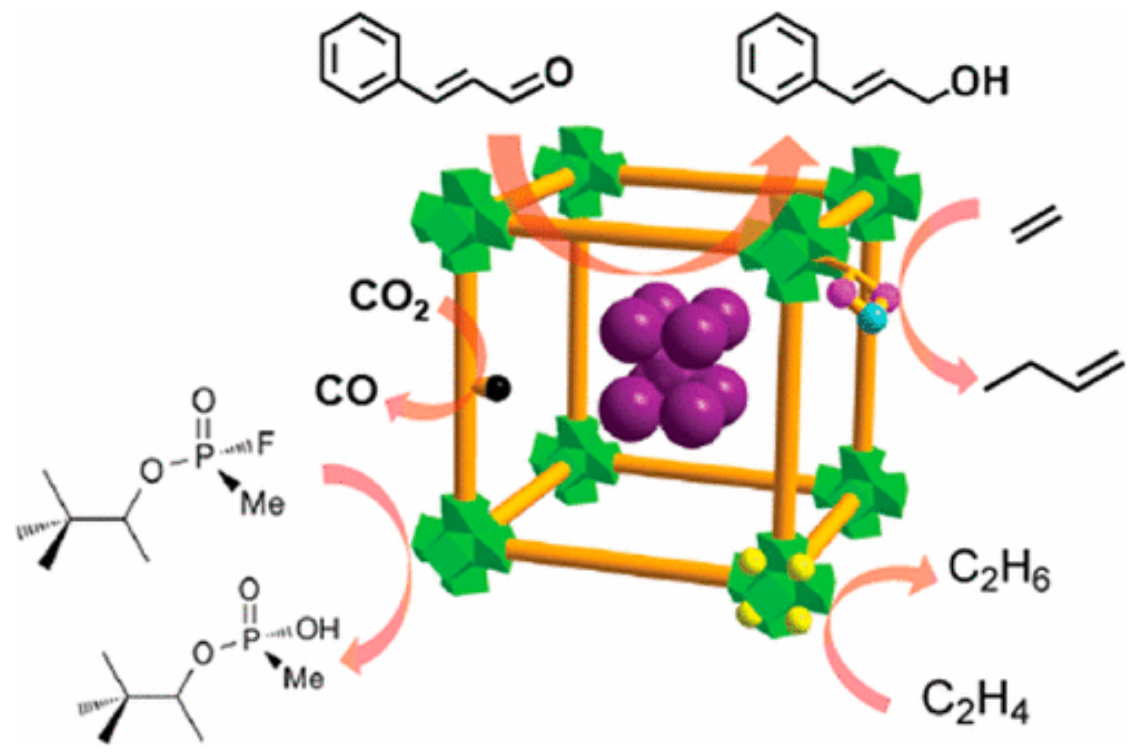

Figure 24. Multifunctional supramolecular $\mathrm{Zr}(\mathrm{Hf})-\mathrm{MOFs}$ catalyst. Reproduced with permission from ref. [72].

Regarding MOCs, assembled MOC motifs such as metallomacrocycles, helical and cylindrical coordination polymers, vertical arrays of 1D coordination polymers, interweaving and eclipsed stacking of 2D coordination polymers have been reported [69]. These materials are photoresponsive systems able to trap radicals, promote photocyclopropanation, include dyes, participate in energy transfer reactions, and reorganize guest-to-host interactions.

\section{Supramolecular catalysis with related inorganic structures}

Supramolecular association between organic compounds and inorganic solids results in hybrid composites with new functionalities and properties in catalysis, photocatalysis and electrocatalysis. There are numerous examples reporting the activity of these organic-inorganic composites in photocatalysis, photovoltaics and as optoelectronic materials in general. As an 
example, the association of terpyridines and inorganic nanoparticles are suitable for applications in nanoelectronics and catalysis due to their stability and the efficiency of EnT and ET processes [73]. One field in which these systems have been applied is in dye-sensitized photoelectrochemical cells (DSSCs-PECs). These types of materials are also considered promising for the production of solar fuels by splitting water into energy-rich hydrogen and oxygen. The photoresponsive composite is based on the assembly of ruthenium polypyridyl complexes with wide bandgap semiconductors. The system may also comprise some polymer as dispersing agent to favor attachment of $\mathrm{TiO}_{2}$ NPs on the electrode (Figure 25) [74].

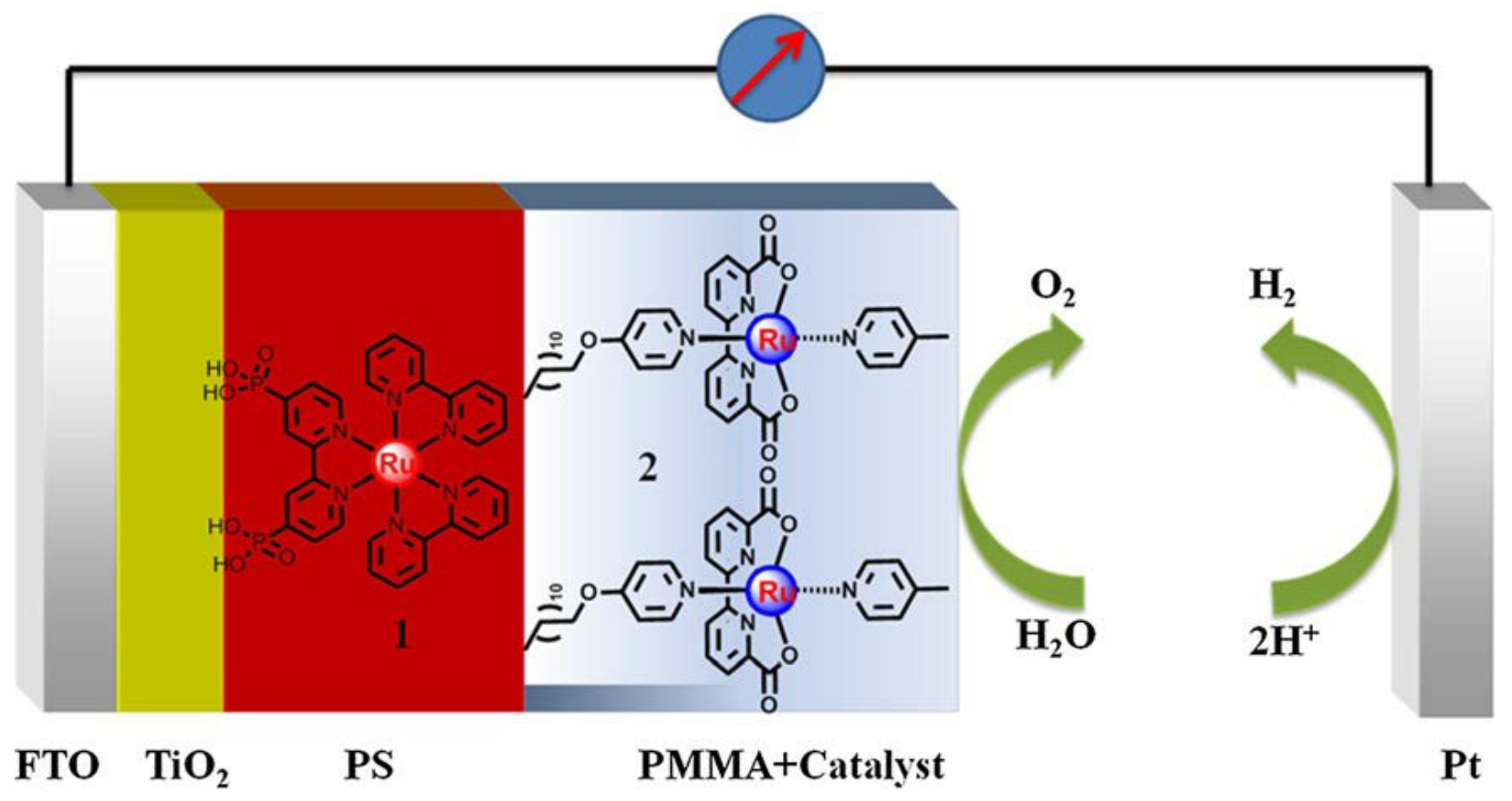

Figure 25. Schematic illustration of supramolecular DSSC-PEC for visible-light driven water splitting in aqueous solution Reproduced with permission from ref. [74]. PS and PMMA refer to polystyrene and poly(methyl methacrylate), respectively. 
A more elaborated supramolecular catalyst of this type is that produced by assembling a coaxial nanohybrid system based on a SWCNT/fullerodendron/SiO 2 nanocomposite (Figure 26) [75]. This system has demonstrated photocatalytic activity for water splitting upon visible light irradiation (450 nm). Under these conditions the quantum yield was of $31 \%$.
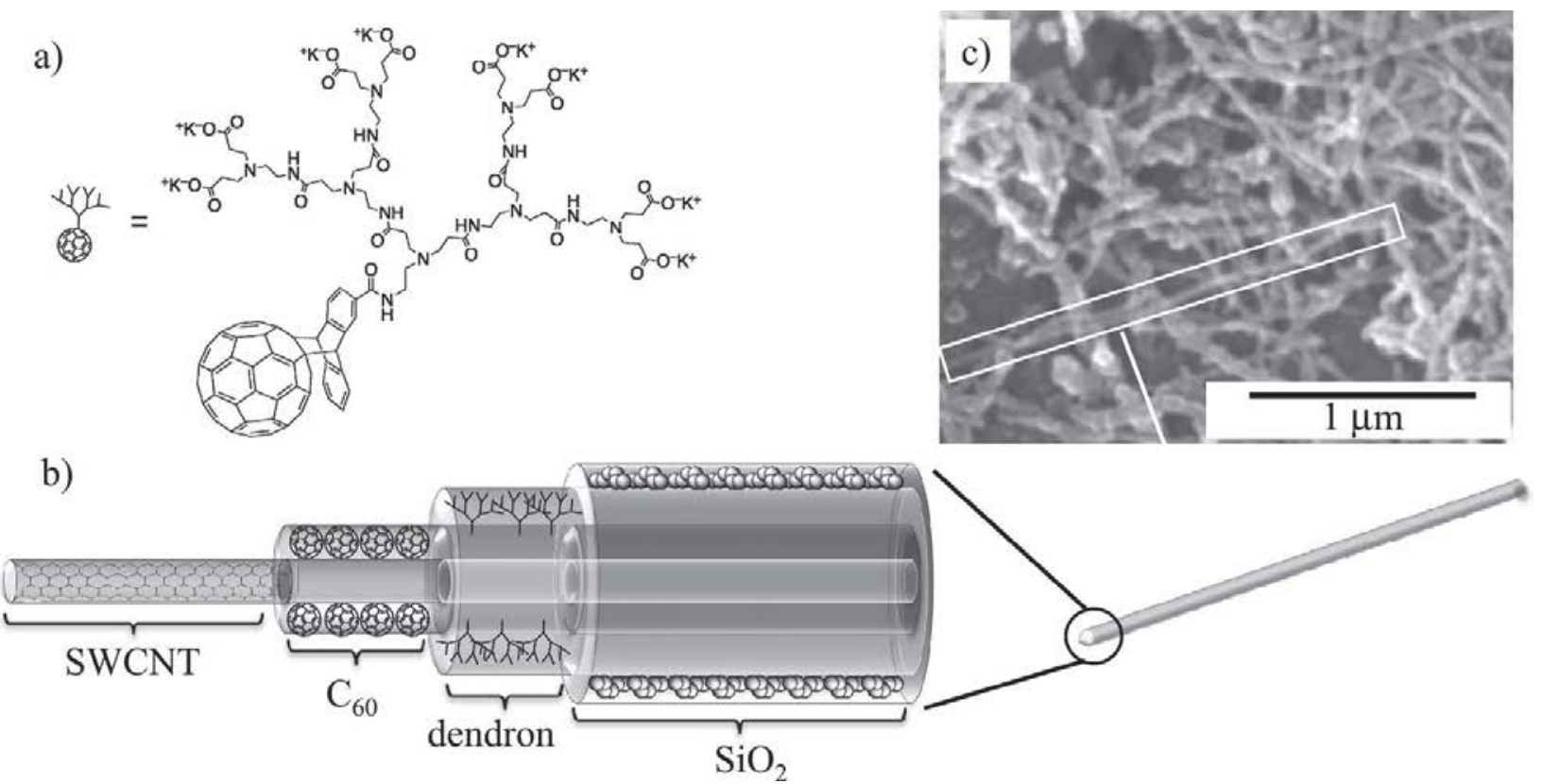

Figure 26. a) Molecular structure of fullerodendron, b) schematic illustration, and c) SEM image of SWCNT/fullerodendron/SiO 2 coaxial nanohybrid. Reproduced with permission from ref. [75].

Periodic mesoporous organosilica (PMO) have also been considered as supports for the production of supramolecular structures. A photocatalytic PMO system for $\mathrm{CO}_{2}$ reduction exhibiting visible-light harvesting has been reported [76]. The mesoporous material consists of a binuclear complex $\left(\mathrm{Ru}^{\mathrm{II}}-\mathrm{Re}^{\mathrm{I}}\right)$ with methylphosphonic acid anchoring groups adsorbed onto the channels of a PMO having disilyl acridone or disilyl methylacridone units forming part of the walls 
of the periodic mesoporous hybrid Figure 27 [76]. The numerous acridone chromophores absorb visible light, and the excitation energy is efficiently funneled to the catalytically active $\mathrm{Ru}^{\mathrm{II}}-\mathrm{Re}^{\mathrm{I}}$ units. This energy accumulation results in intramolecular electron transfer and catalytic reduction of $\mathrm{CO}_{2}$ to $\mathrm{CO}$ on the Re unit. The highly efficient light harvesting ability of these hybrids is a consequence of the high density of acridone units. This high density of chromophores enhances the photocatalytic $\mathrm{CO}$ evolution rate by a factor of up to ten compared with that of the same $\mathrm{Ru}^{\mathrm{II}}$ $\mathrm{Re}^{\mathrm{I}}$ complex adsorbed on mesoporous silica lacking any light harvester. In a certain way the system resembles the situation occurring in natural photosynthesis, where the photosynthetic centers are surrounded by porphyrins as light harvesters.

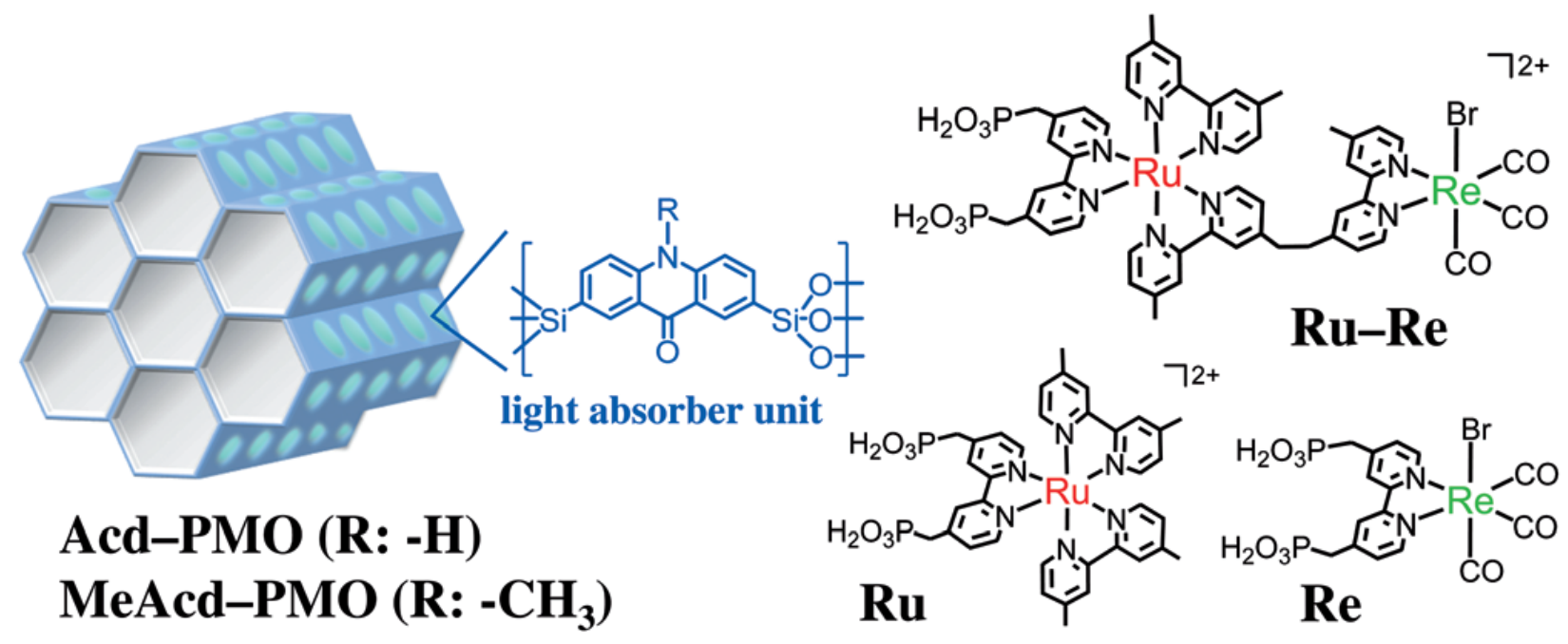

Figure 27. Schematic representation of acridone-PMO embedding $\mathrm{Ru}^{\mathrm{II}}-\mathrm{Re}^{\mathrm{I}}$ complexes that is a system to promote the photocatalytic $\mathrm{CO}_{2}$ reduction. Reproduced with permission from ref. [76]. 
This approach based on selective and directional intermolecular interactions has also been extended to active oxides and metal NPs. These multicomponent PMOs exhibit a remarkable activity enhancement as consequence of the assembly. Formation of supramolecular nanostructures using molecular or polymeric building blocks and NPs require the controlled formation of surface-supported architectures $[77,78]$. The catalytic behavior of these structures can be illustrate with several examples.

Thus, a supramolecular electron-conducting polymer-PtO 2 catalyst showed an unusually low light-off temperatures and high stability in CO oxidation. This high stability is maintained even in the presence of Pt poisons coexisting in practical feedstocks as consequence of the interaction between the active $\mathrm{PtO}_{2}$ and the polymer [79]. Figure 28 illustrates this system.

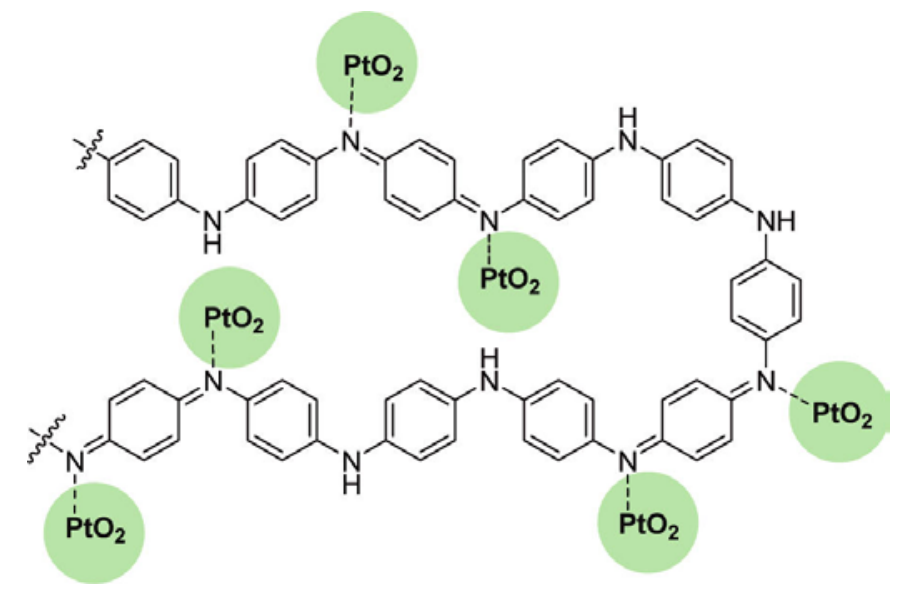

Figure 28. $\mathrm{PtO}_{2} \mathrm{NPs}$ interacting with an electron-conducting polymer, resulting in an enhanced catalyst. Reproduced with permission from ref. [79].

Regarding the metals, Au is among the most common metals for the preparation of biosensors. The (111) plane of Au films can serve as platform to build a flexible porous network via selective supramolecular assembly of this metal with trans-BCaTBPP $[\alpha, \beta$-bis(4- 
carboxyphenyl)- $\beta, \gamma$-bis(3,5-ditertbuylphenyl)porpyrin (see structure in Figure 29), forming wires. Subsequent adsorption of C60 molecules leads to the formation of nanoporous structures. A linear array of opened nanopores is formed by a lateral shift of the supramolecular wires, which is accompanied by a partial change in the conformation of trans-BCaTBPP (Figure 29) [80]. The opening of the nanopores results from the subtle balance between the Au substrate-molecule, on one hand, and the molecule-molecule interactions, on the other. This process controls the formation of flexible porous networks and allows the selective accommodation of guest molecules fitting into the pores. Therefore, in a certain way, the system Au surface-BCaTBPP provide a new approach for molecular recognition and selective catalysis.
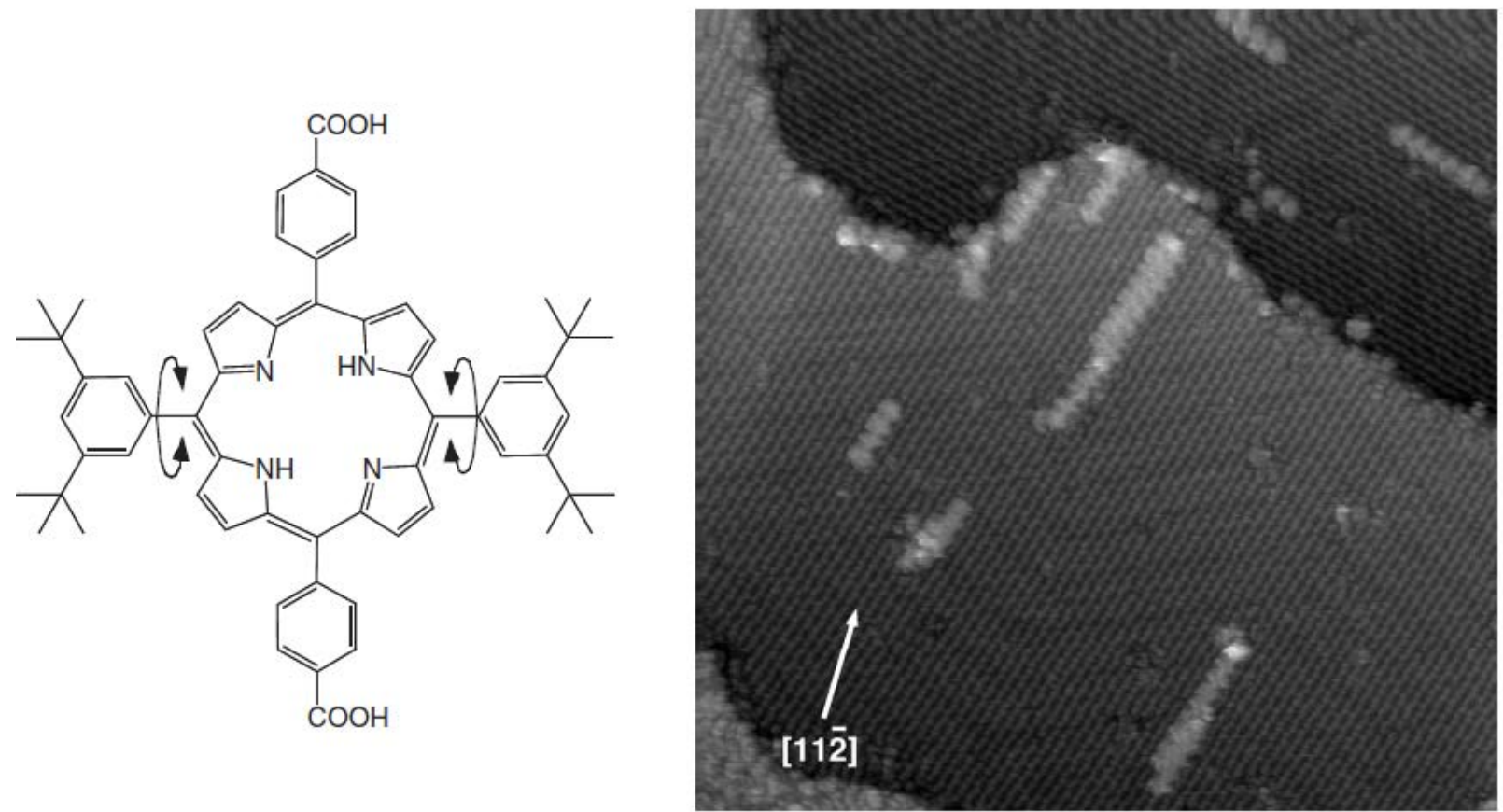

Figure 29. Chemical structure of trans-BCaTBPP and STM image $(120.0 \mathrm{~nm} \times 120.0 \mathrm{~nm}, T=63$

K) of a full-coverage monolayer on $\mathrm{Au}(111)$. With permission from ref. [80]. 
Supramolecular chemistry can be also involved at the stage of catalyst preparation. An example of this possibility is the self-assembly and supramolecular preorganization of the reagents that upon precipitation from the solution and calcination under inert atmosphere at temperatures about $550{ }^{\circ} \mathrm{C}$ renders the corresponding hollow carbon nitride structures (Figure 30). Cyanuric acid forms up to six complementary hydrogen bonds with melanine and these two complementary hydrogen donor-acceptor pair exhibit a strong pre-association in solution leading to a supramolecular structure. Since these two compounds, upon heating, condense to form $\mathrm{C}_{3} \mathrm{~N}_{4}$, it happens that this aggregation leads to the formation of hollowed cyanuric acid-melamine (CM)$\mathrm{C}_{3} \mathrm{~N}_{4}$ structures, when these two molecules precipitate in methanol and then are pyrolyzed under inert atmosphere [81]. These structures exhibit a superior photocatalytic activity in the degradation of the rhodamine B dye. The degradation mechanism was shown to proceed via a photoinduced hole transfer. The improved efficiency of $(\mathrm{CM})-\mathrm{C}_{3} \mathrm{~N}_{4}$ compared to the bulk material was attributed to the structural features of the semiconducting $\mathrm{C}_{3} \mathrm{~N}_{4}$ that arise from this supramolecular preorganization of the precursors.

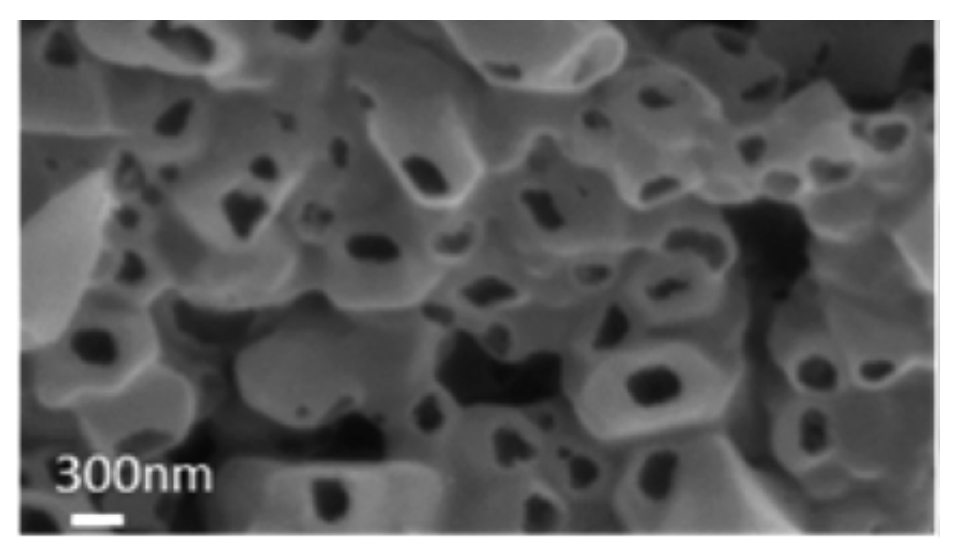

Figure 30. Cyanuric acid-melamine derived hollowed $\mathrm{C}_{3} \mathrm{~N}_{4}$ structures. Reproduced with permission from ref. [81]. 
This concept has been further developed by implementing a sequential solvent treatment of supramolecular solid aggregates that serve as precursors of $\mathrm{C}_{3} \mathrm{~N}_{4}$ [82]. The morphology of cyanuric acid-melamine aggregates and as consequence the morphology, photophysical and catalytic properties of the resulting $\mathrm{C}_{3} \mathrm{~N}_{4}$ semiconductor is strongly dependent on the solvent in which the supramolecular cyanuric acid-melamine is formed [83]. For this reason, stirring the solid before pyrolysis in a second solvent may alter deeply or slightly the supramolecular interactions present in the supramolecular cyanuric acid-melamine aggregates. These aggregates will condense later upon heating in the formation of differently ordered $\mathrm{C}_{3} \mathrm{~N}_{4}$-like materials exhibiting different activity. In this way, stirring the cyanuric acid-melamine solid in a second solvent, depending on its polarity, can lead either to a full rearrangement of the supramolecular cyanuric acid-melamine aggregates or to slight modification of the aggregate surface, still preserving the bulk morphology. This occurs via the creation of different supramolecular crystals along the assembly. The assembly is governed by the hydrogen bond strength across the supramolecular complex causing a difference in the reactivity of inner and the outer crystal upon heating at high temperatures.

Supramolecular chemistry also opens the doors for the synthesis of chiral polyoxometalates (POMs) with potential for chiral asymmetric catalytic oxidation [84]. Examples of enantiomerically pure POM-organic hybrid chiral molecular rods are those assembled from two types of POMs, Lindqvist and Anderson, through linkages with a bifunctional organic ligand (Figure 31). Besides chemical properties, these chiral molecular nanorods nanoarchitectures incorporating rotatable covalent bonds have additional important features, including ferroelectric and non-linear optic properties [84]. 


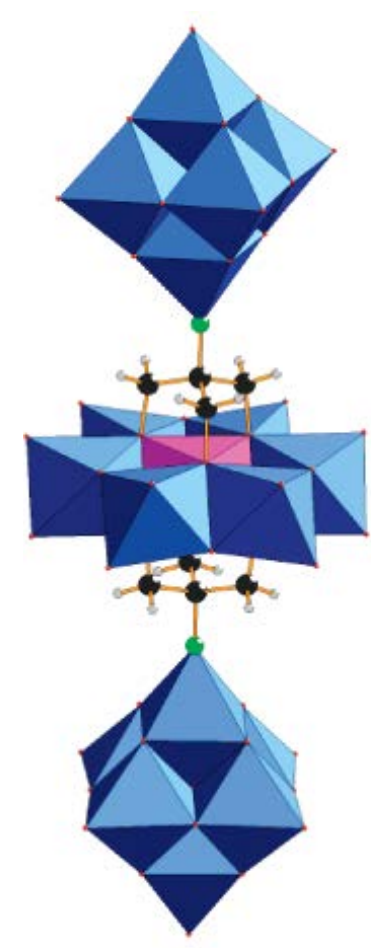

(a)

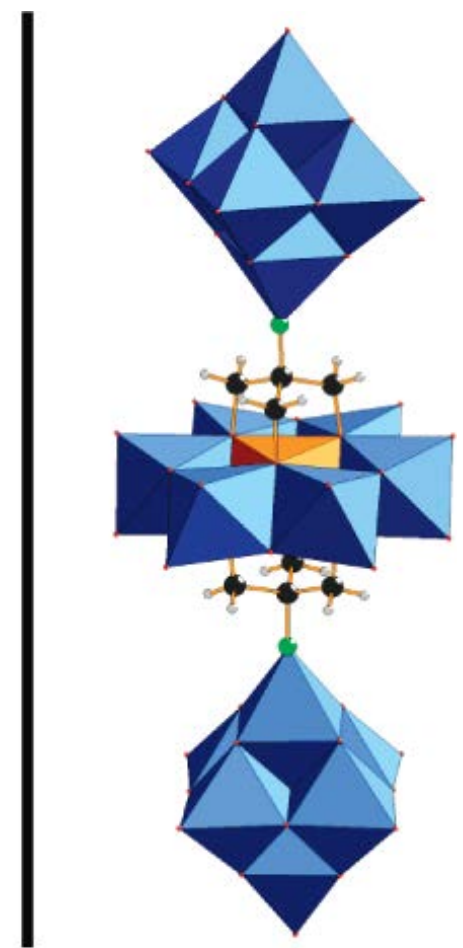

(b)

Figure 31. Examples of chiral polyoxometalates $\left[\mathrm{Mo}_{6} \mathrm{O}_{18} \mathrm{NC}\left(\mathrm{OCH}_{2}\right)_{3} \mathrm{MMo}_{6} \mathrm{O}_{18^{-}}\right.$ $\left.\left(\mathrm{OCH}_{2}\right)_{3} \mathrm{CNMo}_{6} \mathrm{O}_{18}\right]_{7-}(\mathrm{M})=\mathrm{Mn}(\mathrm{III})$, or Fe(III) ions, three and a half cations of $\left(\mathrm{Bu}_{4} \mathrm{~N}\right)^{+}$. Reprinted with permission from ref. [84].

\section{Two-dimensional supramolecular polymers}

Two-dimensional supramolecular polymers (2DSPs), which refer to non-covalently linked networks of monomers, represent another type of supramolecular catalysts. There are in the literature several reported strategies for the synthesis of these highly conductive $\pi$-conjugated 2D nanosheets and various applications have been envisaged [85-87]. These 2D sheets can roll forming tubes [87]. In the case of phenyl-capped bithiophene units connected by a oligoethyleneglycol thread, the linear polymer folds perpendicular to the plane in which the bithiophene units establish supramolecular $\pi-\pi$ interactions [85]. 
Figure 32 depicts a $\pi$-conjugated nanosheet comprising planar nickel bis(dithiolene) complexes. The 2D material with a thickness of several micrometers exhibits semiconducting properties [88]. On this basis, photocatalytic applications of these materials are expected.

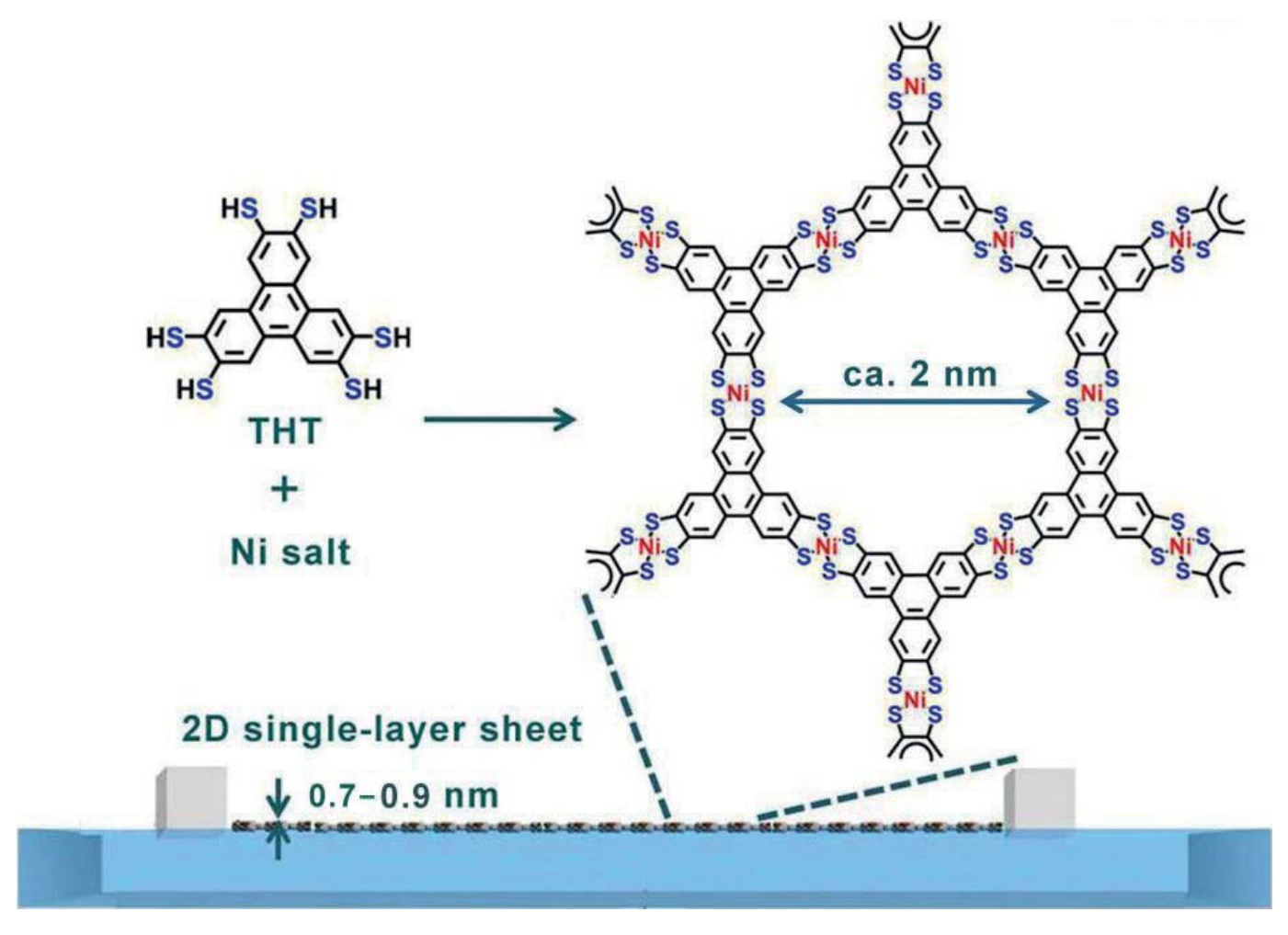

Figure 32. Synthesis of a 2D single-layer sheet composed of triphenylene-fused nickel bis(dithiolene) complexes by using the Langmuir-Blodgett method at an air/water interface. Reprinted with permission from ref. [88].

Polyoxometalate inorganic-organic hybrid structures analogous to those previously commented forming chiral linear rods may also generate 2D supramolecular arrangements when a supramolecular net of aromatic compounds host them. An example is the solid hybrid material 
$\left(\mathrm{H}_{3 / 4} \mathrm{pbpy}_{4}\left[\mathrm{PMo}_{12} \mathrm{O}_{40}\right] 1.25 \mathrm{H}_{2} \mathrm{O}\right.$ (pbpy 5-phenyl-2-(4-pyridinyl)pyridine) Figure 33 [89]. In these case there is a dense packing of the aromatic component, establishing aromatic-aromatic interactions between the pbpys. This result in a compact supramolecular framework structure with suitable nests to accommodate the polyoxometalate units. The distance among the neighbor polyoxometalate is short enough to allow them to establish week $\mathrm{O} \cdots \mathrm{O}$ interactions that also assists the formation of the supramolecular structure. The Keggin molybdate anion in these structures preserves the electrocatalytic activity in several reactions, like electrochemical reduction of chlorate, hydrogen peroxide and nitrite. As consequence of the immobilization of the Keggin heteropolyanion in the organic framework, the supramolecular structure presents better stability than conventional polyoxometallate. Therefore, this supramolecular system is more suitable for practical applications. This improved stability was attributed to the extensive hydrogen bonding and aryl packing interactions in the 2D supramolecular arrangements. 


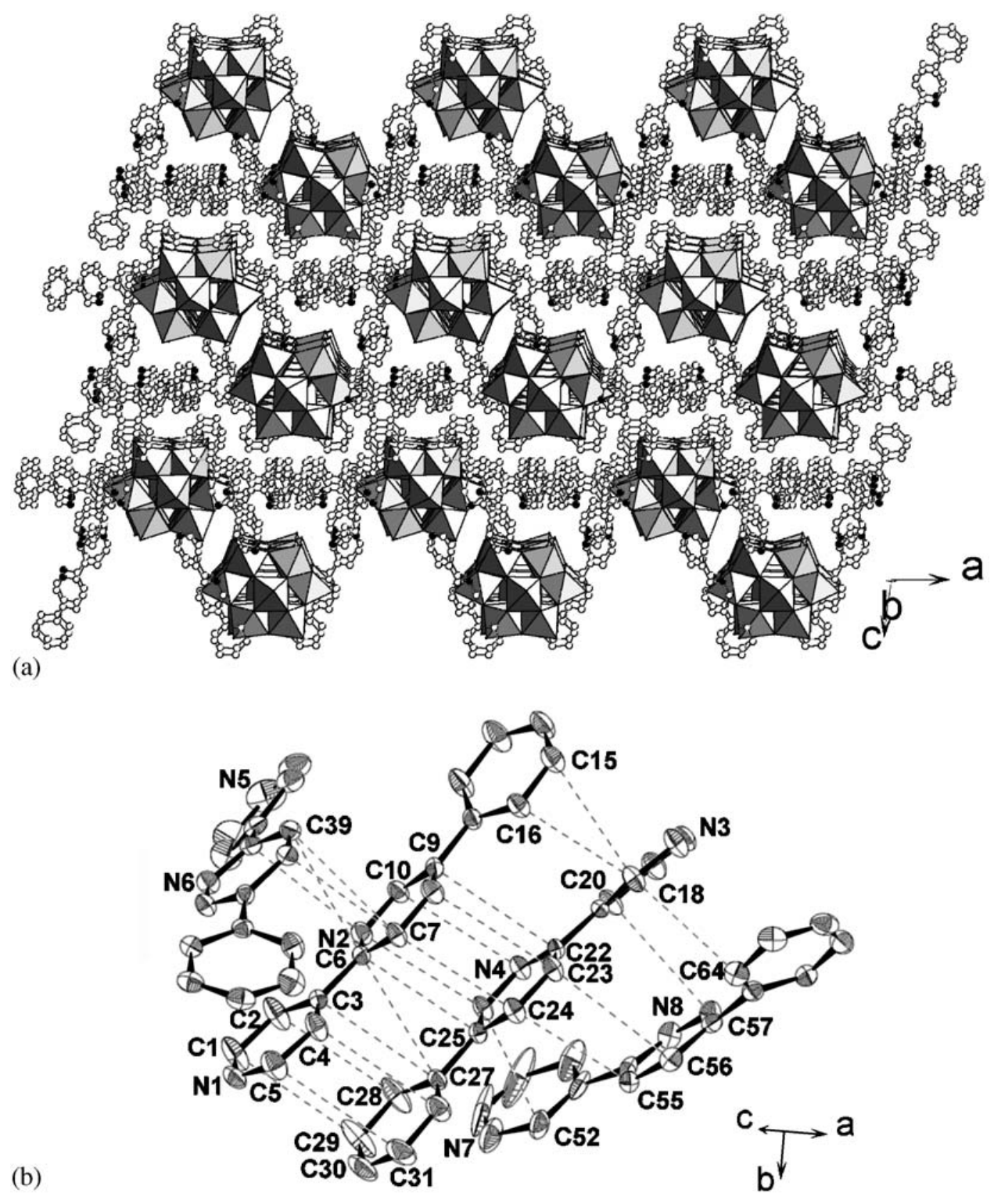

Figure 33. (a) A view of the packing structure along the b axis illustrating the compact packing of pbpy units to nest $\mathrm{PMo}_{12} \mathrm{O}_{40}{ }^{3-}$ clusters. (b) Detail of the packing map of organic molecules establishing $\pi-\pi$ interaction (dotted lines) with distances between 3.503 and $3.888 \AA$. Reprinted with permission from ref. [89]. 


\section{Dynamic supramolecular polymers}

Dendritic polymers containing rotaxane units are a new type of mechanically bonded supramolecules that combine the characteristics of both rotaxanes (sliding and rotary motion) and dendrimers (repetitive branching with each generation) (Figure 34). They offer new opportunities to generate new topological features, particularly suitable to develop electroresponsive materials. The operation mechanism is generally based on the change in the position of the bipyridyl units depending on their charge [90]. For example, functional rotaxane dendrimers substituted with ferrocenes as termini may provide a platform for investigations of these molecules in molecular electronics [90]. These dendritic polyrotaxenes can be enantiomerically pure. Their use as asymmetric ligands may allow developing the heterogeneous versions of numerous asymmetric reactions that are efficient under homogeneous phase. In this way the supramolecular system possesses the multiple advantages derived from the recovery of the catalyst and its reusability.
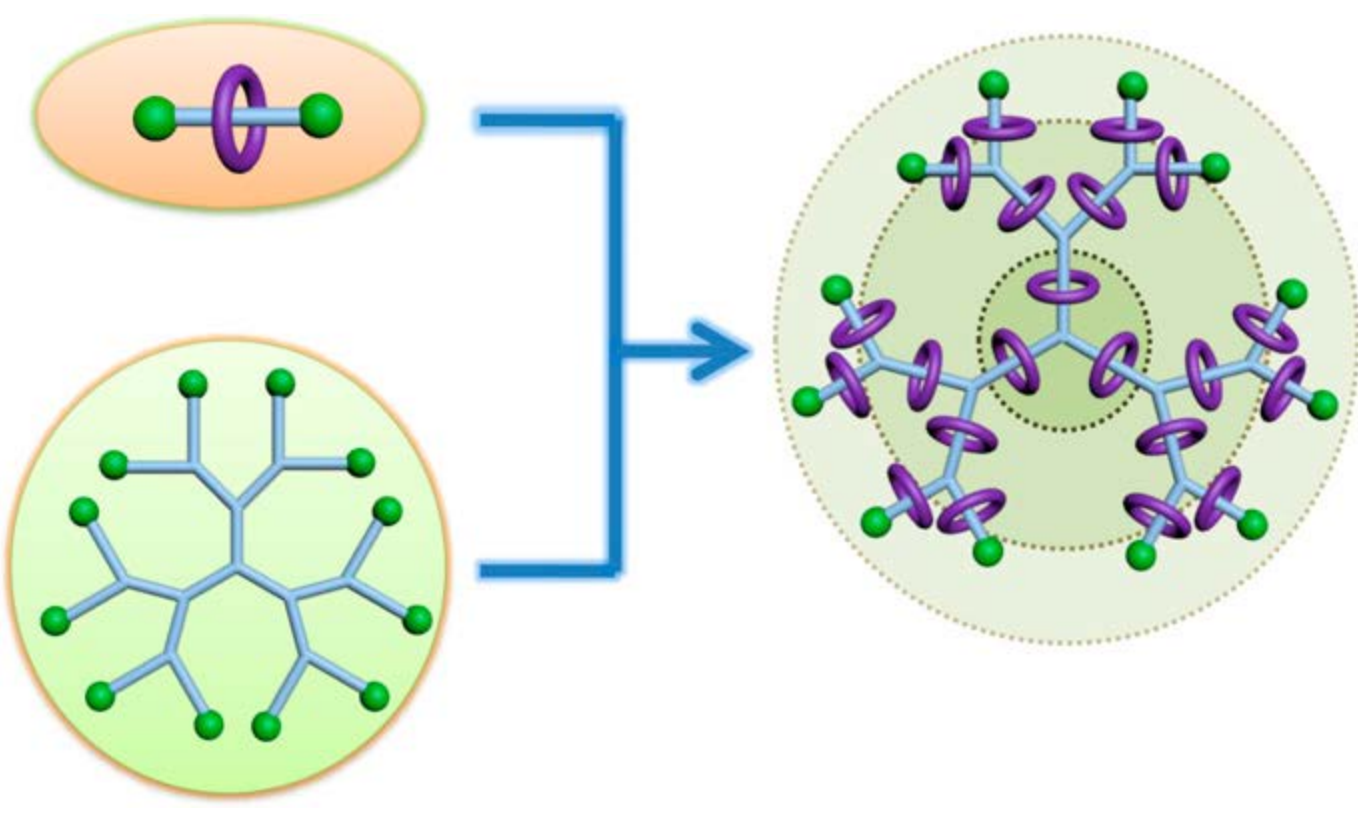
Figure 34. Schematic representation of a rotaxane dendrimer with mechanically interlocked moieties incorporated on the branches. Reprinted with permission from ref. [90].

\section{Conclusions.}

The present article has illustrated the potential that the use of $\mathrm{sp}^{2}$ carbon sheets, either graphene or carbon nanotubes, offer as platform to anchor through van der Waals interactions units that have catalytic activity. These units can be organic molecules, metal complexes and metallic nanoparticles. This approach has been well developed. There are numerous examples showing the advantages derived from the easy immobilization procedure and the synergy between the carbon material and the active components. Additional examples should expand the area showing the synergy that can arise from the use of the carbon material as compared to other supports. The origin of this synergy can derive from the electronic properties, adsorption capacity and the combination of the intrinsic catalytic activity of defective graphenes [91]. Application of these supramolecular assemblies appear to be specially suited in the in the area of biocatalysis, where enzymes can be wrapped by flexible carbon sheets, while still allowing electron transfer and substrate diffusion.

Using carbon nitride as example, the possibility to use supramolecular aggregation in the preparation of a solid catalyst with tunable physicochemical and catalytic properties has been presented. It has been discussed how the catalytic activity of $\mathrm{C}_{3} \mathrm{~N}_{4}$ varies depending on the solvent used to disrupt or promote hydrogen bonding between cyanuric acid and melamine. It is clear that these concepts can lay behind many recipes of materials preparation. However, the conscious manipulation of pre-aggregation of the components is very frequently not fully exploited to enhance catalytic activity. 
It is clear that the field of supramolecular catalysis is not limited to graphene and carbon nanotubes. There are multitude of organic molecules that either through $\pi-\pi$ interactions or by metal-ligand coordinative bonds can construct supramolecular assemblies with potential in catalysis. The list includes photo- and electroactive molecular machines that through their movement can shuttle electrons from to chemical substrate the working electrode.

Supramolecular aggregation in catalysis may take the advantage of two structural characteristics. Firstly, the involvement of the $\pi$ electrons in the construction of these entities may tune the behavior of these materials in heterogeneous catalysis. This tuning can be of special relevance in the case of metal nanoparticles and in electrocatalysis. The changes in the band gap can also influence the photocatalytic reactions. On the other side, aggregation may control reactions like those catalyzed by acid and bases and, with the recent achievements, asymmetric reactions as well.

In view of the present state of the art, it can be easily forecast that the use of supramolecular aggregation in catalysis will increase in the near future, particularly, but not only for graphenes and carbon nanotubes. Applying concept of supramolecular aggregation may simplify longer routes based on the formation of covalent bonds and offer new opportunities for the preparations of novel catalysts.

\section{Acknowledgements.}

Financial support by the Spanish Ministry of Economy and Competitiveness (Severo Ochoa and CTQ2015-69153-CO2-R1) and Generalitat Valenciana (Prometeo 2017-083) is gratefully acknowledged. 


\section{References.}

[1] J.-M. Lehn, Supramolecular chemistry, Vch, Weinheim1995.

[2] J.W. Steed, J.L. Atwood, P.A. Gale, Definition and emergence of supramolecular chemistry, Wiley Online Library2012.

[3] S. Herbst, B. Soberats, P. Leowanawat, M. Stolte, M. Lehmann, F. Würthner, Self-assembly of multistranded perylene dye J-aggregates in columnar liquid-crystalline phases, Nature communications, 9 (2018) 2646.

[4] F. Würthner, C. Thalacker, A. Sautter, Hierarchical organization of functional perylene chromophores to mesoscopic superstructures by hydrogen bonding and $\pi-\pi$ interactions, Advanced Materials, 11 (1999) 754-758.

[5] E.E. Jelley, Spectral absorption and fluorescence of dyes in the molecular state, Nature, 138 (1936) 1009.

[6] J. Wang, D. Liu, Y. Zhu, S. Zhou, S. Guan, Supramolecular packing dominant photocatalytic oxidation and anticancer performance of PDI, Applied Catalysis B: Environmental, 231 (2018) 251-261.

[7] P. Liebing, E. Pietrasiak, E. Otth, J. Kalim, D. Bornemann, A. Togni, Supramolecular Aggregation of Perfluoroorganyl lodane Reagents in the Solid State and in Solution, European Journal of Organic Chemistry, 2018 (2018) 3771-3781.

[8] S. Zhang, Fabrication of novel biomaterials through molecular self-assembly, Nature biotechnology, 21 (2003) 1171-1178.

[9] V. Balzani, M. Gómez-López, J.F. Stoddart, Molecular machines, Accounts of Chemical Research, 31 (1998) 405-414.

[10] C. Bai, M. Liu, Implantation of nanomaterials and nanostructures on surface and their applications, Nano Today, 7 (2012) 258-281.

[11] J.-M. Lehn, Toward complex matter: Supramolecular chemistry and self-organization, Proceedings of the National Academy of Sciences, 99 (2002) 4763-4768.

[12] J.-M. Lehn, From supramolecular chemistry towards constitutional dynamic chemistry and adaptive chemistry, Chemical Society Reviews, 36 (2007) 151-160.

[13] J.K. Sanders, Supramolecular catalysis in transition, Chemistry-A European Journal, 4 (1998) 13781383.

[14] A. Lützen, Supramolecular Catalysis. Edited by Piet WNM van Leeuwen, Wiley Online Library, 2008.

[15] L. Zhao, X.-L. Sui, J.-Z. Li, J.-J. Zhang, L.-M. Zhang, G.-S. Huang, Z.-B. Wang, Supramolecular assembly promoted synthesis of three-dimensional nitrogen doped graphene frameworks as efficient electrocatalyst for oxygen reduction reaction and methanol electrooxidation, Applied Catalysis B: Environmental, 231 (2018) 224-233.

[16] X. Wang, Q. Liu, Q. Yang, Z. Zhang, X. Fang, Three-dimension g-C3N4 aggregate composed of hollow bubbles with high activity for photocatalytic degradation of tetracycline, Carbon, 136 (2018) 103-112.

[17] Y. Yao, X. Wei, Y. Cai, X. Kong, J. Chen, J. Wu, Y. Shi, Hybrid supramolecular materials constructed from pillar [5] arene based host-guest interactions with photo and redox tunable properties, Journal of colloid and interface science, 525 (2018) 48-53.

[18] F.C.-M. Leung, S.Y.-L. Leung, C.Y.-S. Chung, V.W.-W. Yam, Metal-metal and $\pi-\pi$ interactions directed end-to-end assembly of gold nanorods, Journal of the American Chemical Society, 138 (2016) 2989-2992. [19] C. Lu, M. Zhang, D. Tang, X. Yan, Z. Zhang, Z. Zhou, B. Song, H. Wang, X. Li, S. Yin, H. Sepehrpour, P.J. Stang, Fluorescent Metallacage-Core Supramolecular Polymer Gel Formed by Orthogonal Metal Coordination and Host-Guest Interactions, Journal of the American Chemical Society, 140 (2018) 76747680.

[20] Y. Sun, S. Li, Z. Zhou, M.L. Saha, S. Datta, M. Zhang, X. Yan, D. Tian, H. Wang, L. Wang, X. Li, M. Liu, H. Li, P.J. Stang, Alanine-Based Chiral Metallogels via Supramolecular Coordination Complex Platforms: 
Metallogelation Induced Chirality Transfer, Journal of the American Chemical Society, 140 (2018) 32573263.

[21] P. Du, M. Jaouen, A. Bocheux, C. Bourgogne, Z. Han, V. Bouchiat, D. Kreher, F. Mathevet, C. FioriniDebuisschert, F. Charra, Surface-Confined Self-Assembled Janus Tectons: A Versatile Platform towards the Noncovalent Functionalization of Graphene, Angewandte Chemie, 126 (2014) 10224-10230.

[22] V. Georgakilas, M. Otyepka, A.B. Bourlinos, V. Chandra, N. Kim, K.C. Kemp, P. Hobza, R. Zboril, K.S. Kim, Functionalization of graphene: covalent and non-covalent approaches, derivatives and applications, Chemical reviews, 112 (2012) 6156-6214.

[23] S. Qu, M. Li, L. Xie, X. Huang, J. Yang, N. Wang, S. Yang, Noncovalent functionalization of graphene attaching $[6,6]$-phenyl-C61-butyric acid methyl ester (PCBM) and application as electron extraction layer of polymer solar cells, ACS nano, 7 (2013) 4070-4081.

[24] P. Du, D. Bléger, F. Charra, V. Bouchiat, D. Kreher, F. Mathevet, A.-J. Attias, A versatile strategy towards non-covalent functionalization of graphene by surface-confined supramolecular self-assembly of Janus tectons, Beilstein journal of nanotechnology, 6 (2015) 632.

[25] B. Chefetz, A.P. Deshmukh, P.G. Hatcher, E.A. Guthrie, Pyrene sorption by natural organic matter, Environmental science \& technology, 34 (2000) 2925-2930.

[26] B. Pan, B. Xing, Adsorption mechanisms of organic chemicals on carbon nanotubes, Environmental science \& technology, 42 (2008) 9005-9013.

[27] J. Chen, W. Chen, D. Zhu, Adsorption of nonionic aromatic compounds to single-walled carbon nanotubes: effects of aqueous solution chemistry, Environmental science \& technology, 42 (2008) 72257230.

[28] R. Podeszwa, Interactions of graphene sheets deduced from properties of polycyclic aromatic hydrocarbons, The Journal of chemical physics, 132 (2010) 044704.

[29] E. Peris, Polyaromatic N-heterocyclic carbene ligands and $\pi$-stacking. Catalytic consequences, Chemical Communications, 52 (2016) 5777-5787.

[30] S. Ruiz-Botella, E. Peris, Unveiling the Importance of $\pi$-Stacking in Borrowing-Hydrogen Processes Catalysed by Iridium Complexes with Pyrene Tags, Chemistry-A European Journal, 21 (2015) 15263-15271. [31] S. Sabater, J.A. Mata, E. Peris, Immobilization of pyrene-tagged Palladium and Ruthenium complexes onto reduced graphene oxide: an efficient and highly recyclable catalyst for hydrodefluorination, Organometallics, 34 (2014) 1186-1190.

[32] S. Sabater, J.A. Mata, E. Peris, Catalyst enhancement and recyclability by immobilization of metal complexes onto graphene surface by noncovalent interactions, Acs Catalysis, 4 (2014) 2038-2047.

[33] S. Wittmann, A. Schätz, R.N. Grass, W.J. Stark, O. Reiser, A Recyclable Nanoparticle-Supported Palladium Catalyst for the Hydroxycarbonylation of Aryl Halides in Water, Angewandte Chemie International Edition, 49 (2010) 1867-1870.

[34] M. Keller, V. Collière, O. Reiser, A.M. Caminade, J.P. Majoral, A. Ouali, Pyrene-Tagged Dendritic Catalysts Noncovalently Grafted onto Magnetic Co/C Nanoparticles: An Efficient and Recyclable System for Drug Synthesis, Angewandte Chemie International Edition, 52 (2013) 3626-3629.

[35] S. Mishra, S. Arora, R. Nagpal, S.M.S. Chauhan, Sulfonated graphenes catalyzed synthesis of expanded porphyrins and their supramolecular interactions with pristine graphene, Journal of Chemical Sciences, 126 (2014) 1729-1736.

[36] L. Xing, J.H. Xie, Y.S. Chen, L.X. Wang, Q.L. Zhou, Simply Modified Chiral Diphosphine: Catalyst Recycling via Non-covalent Absorption on Carbon Nanotubes, Advanced Synthesis \& Catalysis, 350 (2008) 1013-1016.

[37] G. Che, B.B. Lakshmi, E.R. Fisher, C.R. Martin, Carbon nanotubule membranes for electrochemical energy storage and production, Nature, 393 (1998) 346.

[38] Z. Zhu, D. Su, G. Weinberg, R. Schlögl, Supermolecular self-assembly of graphene sheets: Formation of tube-in-tube nanostructures, Nano Letters, 4 (2004) 2255-2259. 
[39] T. Fukushima, A. Kosaka, Y. Ishimura, T. Yamamoto, T. Takigawa, N. Ishii, T. Aida, Molecular ordering of organic molten salts triggered by single-walled carbon nanotubes, Science, 300 (2003) 2072-2074.

[40] M. Tunckol, J. Durand, P. Serp, Carbon nanomaterial-ionic liquid hybrids, Carbon, 50 (2012) 43034334.

[41] K. Subramaniam, A. Das, G. Heinrich, Development of conducting polychloroprene rubber using imidazolium based ionic liquid modified multi-walled carbon nanotubes, Composites Science and Technology, 71 (2011) 1441-1449.

[42] H. Chu, Y. Shen, L. Lin, X. Qin, G. Feng, Z. Lin, J. Wang, H. Liu, Y. Li, lonic-Liquid-Assisted Preparation of Carbon Nanotube-Supported Uniform Noble Metal Nanoparticles and Their Enhanced Catalytic Performance, Advanced Functional Materials, 20 (2010) 3747-3752.

[43] Y.S. Chun, J.Y. Shin, C.E. Song, S.-g. Lee, Palladium nanoparticles supported onto ionic carbon nanotubes as robust recyclable catalysts in an ionic liquid, Chemical Communications, (2008) 942-944.

[44] A.M.P. Salvo, V. La Parola, L.F. Liotta, F. Giacalone, M. Gruttadauria, Highly Loaded Multi-Walled Carbon Nanotubes Non-Covalently Modified with a Bis-Imidazolium Salt and their Use as Catalyst Supports, ChemPlusChem, 81 (2016) 471-476.

[45] H.S. Park, B.G. Choi, S.H. Yang, W.H. Shin, J.K. Kang, D. Jung, W.H. Hong, lonic-Liquid-Assisted Sonochemical Synthesis of Carbon-Nanotube-Based Nanohybrids: Control in the Structures and Interfacial Characteristics, Small, 5 (2009) 1754-1760.

[46] S. Noël, B. Léger, A. Ponchel, K. Philippot, A. Denicourt-Nowicki, A. Roucoux, E. Monflier, Cyclodextrinbased systems for the stabilization of metallic (0) nanoparticles and their versatile applications in catalysis, Catalysis Today, 235 (2014) 20-32.

[47] F. Wyrwalski, B. Léger, C. Lancelot, A. Roucoux, E. Monflier, A. Ponchel, Chemically modified cyclodextrins as supramolecular tools to generate carbon-supported ruthenium nanoparticles: An application towards gas phase hydrogenation, Applied Catalysis A: General, 391 (2011) 334-341.

[48] A. Jean-Marie, A. Griboval-Constant, A.Y. Khodakov, E. Monflier, F. Diehl, $\beta$-Cyclodextrin for design of alumina supported cobalt catalysts efficient in Fischer-Tropsch synthesis, Chemical Communications, 47 (2011) 10767-10769.

[49] B. Léger, S. Menuel, A. Ponchel, F. Hapiot, E. Monflier, Nanoparticle-Based Catalysis using Supramolecular Hydrogels, Advanced Synthesis \& Catalysis, 354 (2012) 1269-1272.

[50] J.J. Zhang, J.M. Ge, H.H. Wang, X. Wei, X.H. Li, J.S. Chen, Activating Oxygen Molecules over CarbonylModified Graphitic Carbon Nitride: Merging Supramolecular Oxidation with Photocatalysis in a Metal-Free Catalyst for Oxidative Coupling of Amines into Imines, ChemCatChem, 8 (2016) 3441-3445.

[51] W. Qi, W. Liu, S. Liu, B. Zhang, X. Gu, X. Guo, D. Su, Heteropoly Acid/Carbon Nanotube Hybrid Materials as Efficient Solid-Acid Catalysts, ChemCatChem, 6 (2014) 2613-2620.

[52] B. Willner, E. Katz, I. Willner, Electrical contacting of redox proteins by nanotechnological means, Current opinion in biotechnology, 17 (2006) 589-596.

[53] R.E. Smalley, Y. Li, V.C. Moore, B.K. Price, R. Colorado, H.K. Schmidt, R.H. Hauge, A.R. Barron, J.M. Tour, Single wall carbon nanotube amplification: En route to a type-specific growth mechanism, Journal of the American Chemical Society, 128 (2006) 15824-15829.

[54] R. Jasti, J. Bhattacharjee, J.B. Neaton, C.R. Bertozzi, Synthesis, characterization, and theory of [9],[12]-, and [18] cycloparaphenylene: carbon nanohoop structures, Journal of the American Chemical Society, 130 (2008) 17646-17647.

[55] E.H. Fort, P.M. Donovan, L.T. Scott, Diels- Alder Reactivity of Polycyclic Aromatic Hydrocarbon Bay Regions: Implications for Metal-Free Growth of Single-Chirality Carbon Nanotubes, Journal of the American Chemical Society, 131 (2009) 16006-16007.

[56] E.H. Fort, L.T. Scott, One-Step Conversion of Aromatic Hydrocarbon Bay Regions into Unsubstituted Benzene Rings: A Reagent for the Low-Temperature, Metal-Free Growth of Single-Chirality Carbon Nanotubes, Angewandte Chemie, 122 (2010) 6776-6778. 
[57] D. Lu, S. Cui, P. Du, Large $\pi$-Extension of Carbon Nanorings by Incorporating Hexa-perihexabenzocoronenes, Synlett, 28 (2017) 1671-1677.

[58] T. Niu, J. Wu, F. Ling, S. Jin, G.-H. Lu, M. Zhou, Halogen-adatom Mediated Phase Transition of Twodimensional Molecular Self-assembly on Metal Surface, Langmuir, (2017).

[59] J.W. Lee, S. Samal, N. Selvapalam, H.-J. Kim, K. Kim, Cucurbituril homologues and derivatives: new opportunities in supramolecular chemistry, Accounts of Chemical Research, 36 (2003) 621-630.

[60] X.-L. Ni, X. Xiao, H. Cong, Q.-J. Zhu, S.-F. Xue, Z. Tao, Self-assemblies based on the "outer-surface interactions" of cucurbit [n] urils: new opportunities for supramolecular architectures and materials, Accounts of chemical research, 47 (2014) 1386-1395.

[61] P. Wang, Y. Wu, Y. Zhao, Y. Yu, M. Zhang, L. Cao, Crystalline nanotubular framework constructed by cucurbit [8] uril for selective CO 2 adsorption, Chemical Communications, 53 (2017) 5503-5506.

[62] S.L. James, Metal-organic frameworks, Chemical Society Reviews, 32 (2003) 276-288.

[63] H.-C. Zhou, J.R. Long, O.M. Yaghi, Introduction to metal-organic frameworks, ACS Publications, 2012.

[64] A. Dhakshinamoorthy, A.M. Asiri, H. Garcia, Metal organic frameworks as versatile hosts of Au nanoparticles in heterogeneous catalysis, ACS Catalysis, 7 (2017) 2896-2919.

[65] A. Dhakshinamoorthy, H. Garcia, Cascade reactions catalyzed by metal organic frameworks, ChemSusChem, 7 (2014) 2392-2410.

[66] A. Dhakshinamoorthy, A.M. Asiri, H. Garcia, Catalysis by metal-organic frameworks in water, Chemical Communications, 50 (2014) 12800-12814.

[67] A. Dhakshinamoorthy, A.M. Asiri, H. Garcia, Metal-organic frameworks as catalysts for oxidation reactions, Chemistry-A European Journal, 22 (2016) 8012-8024.

[68] T.H. Noh, O.-S. Jung, Recent Advances in Various Metal-Organic Channels for Photochemistry beyond Confined Spaces, Accounts of chemical research, 49 (2016) 1835-1843.

[69] G. Tabacchi, Supramolecular Organization in Confined Nanospaces, ChemPhysChem, 19 (2018) 12491297.

[70] R. Haldar, S.K. Reddy, V.M. Suresh, S. Mohapatra, S. Balasubramanian, T.K. Maji, Flexible and Rigid Amine-Functionalized Microporous Frameworks Based on Different Secondary Building Units: Supramolecular Isomerism, Selective CO2 Capture, and Catalysis, Chemistry-A European Journal, 20 (2014) 4347-4356.

[71] L.-L. Tan, N. Song, S.X.-A. Zhang, H. Li, B. Wang, Y.-W. Yang, Ca2+, pH and thermo triple-responsive mechanized Zr-based MOFs for on-command drug release in bone diseases, Journal of Materials Chemistry B, 4 (2016) 135-140.

[72] M. Rimoldi, A.J. Howarth, M.R. DeStefano, L. Lin, S. Goswami, P. Li, J.T. Hupp, O.K. Farha, Catalytic zirconium/hafnium-based metal-organic frameworks, ACS Catalysis, 7 (2016) 997-1014.

[73] A. Winter, M.D. Hager, G.R. Newkome, U.S. Schubert, The marriage of terpyridines and inorganic nanoparticles: synthetic aspects, characterization techniques, and potential applications, Advanced Materials, 23 (2011) 5728-5748.

[74] X. Ding, Y. Gao, L. Ye, L. Zhang, L. Sun, Assembling Supramolecular Dye-Sensitized Photoelectrochemical Cells for Water Splitting, ChemSusChem, 8 (2015) 3992-3995.

[75] T. Tajima, W. Sakata, T. Wada, A. Tsutsui, S. Nishimoto, M. Miyake, Y. Takaguchi, Photosensitized Hydrogen Evolution from Water Using a Single-Walled Carbon Nanotube/Fullerodendron/SiO2 Coaxial Nanohybrid, Advanced Materials, 23 (2011) 5750-5754.

[76] Y. Ueda, H. Takeda, T. Yui, K. Koike, Y. Goto, S. Inagaki, O. Ishitani, A Visible-Light Harvesting System for $\mathrm{CO} 2$ Reduction Using a Rull-Rel Photocatalyst Adsorbed in Mesoporous Organosilica, ChemSusChem, 8 (2015) 439-442.

[77] T. Yokoyama, S. Yokoyama, T. Kamikado, Y. Okuno, S. Mashiko, Selective assembly on a surface of supramolecular aggregates with controlled size and shape, Nature, 413 (2001) 619. 
[78] J.V. Barth, G. Costantini, K. Kern, Engineering atomic and molecular nanostructures at surfaces, Nature, $437(2005) 671$.

[79] F. Klasovsky, J. Hohmeyer, A. Brückner, M. Bonifer, J.r. Arras, M. Steffan, M. Lucas, J.r. Radnik, C. Roth, P. Claus, Catalytic and Mechanistic Investigation of Polyaniline Supported PtO2 Nanoparticles: A Combined in situ/operando EPR, DRIFTS, and EXAFS Study, The Journal of Physical Chemistry C, 112 (2008) 19555-19559.

[80] F. Nishiyama, T. Yokoyama, T. Kamikado, S. Yokoyama, S. Mashiko, K. Sakaguchi, K. Kikuchi, Interstitial Accommodation of C60 in a Surface-Supported Supramolecular Network, Advanced Materials, 19 (2007) 117-120.

[81] M. Shalom, S. Inal, C. Fettkenhauer, D. Neher, M. Antonietti, Improving carbon nitride photocatalysis by supramolecular preorganization of monomers, Journal of the American Chemical Society, 135 (2013) 7118-7121.

[82] J. Sun, J. Xu, A. Grafmueller, X. Huang, C. Liedel, G. Algara-Siller, M. Willinger, C. Yang, Y. Fu, X. Wang, Self-assembled carbon nitride for photocatalytic hydrogen evolution and degradation of p-nitrophenol, Applied Catalysis B: Environmental, 205 (2017) 1-10.

[83] Y. Ishida, L. Chabanne, M. Antonietti, M. Shalom, Morphology control and photocatalysis enhancement by the one-pot synthesis of carbon nitride from preorganized hydrogen-bonded supramolecular precursors, Langmuir, 30 (2014) 447-451.

[84] J. Zhang, J. Hao, Y. Wei, F. Xiao, P. Yin, L. Wang, Nanoscale Chiral Rod-like Molecular Triads Assembled from Achiral Polyoxometalates, Journal of the American Chemical Society, 132 (2010) 14-15.

[85] Y. Zheng, H. Zhou, D. Liu, G. Floudas, M. Wagner, K. Koynov, M. Mezger, H.J. Butt, T. Ikeda, Supramolecular thiophene nanosheets, Angewandte Chemie, 125 (2013) 4945-4948.

[86] E. Lee, J.K. Kim, M. Lee, Reversible Scrolling of Two-Dimensional Sheets from the Self-Assembly of Laterally Grafted Amphiphilic Rods, Angewandte Chemie International Edition, 48 (2009) 3657-3660.

[87] T. Kambe, R. Sakamoto, K. Hoshiko, K. Takada, M. Miyachi, J.-H. Ryu, S. Sasaki, J. Kim, K. Nakazato, M. Takata, $\pi$-Conjugated nickel bis (dithiolene) complex nanosheet, Journal of the American Chemical Society, 135 (2013) 2462-2465.

[88] R. Dong, M. Pfeffermann, H. Liang, Z. Zheng, X. Zhu, J. Zhang, X. Feng, Large-Area, Free-Standing, TwoDimensional Supramolecular Polymer Single-Layer Sheets for Highly Efficient Electrocatalytic Hydrogen Evolution, Angewandte Chemie International Edition, 54 (2015) 12058-12063.

[89] Z. Han, Y. Zhao, J. Peng, A. Tian, Y. Feng, Q. Liu, Inorganic-organic hybrid polyoxometalate: Preparation, characterization and electrochemistry properties, Journal of Solid State Chemistry, 178 (2005) 1386-1394.

[90] W. Wang, L.-J. Chen, X.-Q. Wang, B. Sun, X. Li, Y. Zhang, J. Shi, Y. Yu, L. Zhang, M. Liu, Organometallic rotaxane dendrimers with fourth-generation mechanically interlocked branches, Proceedings of the National Academy of Sciences, 112 (2015) 5597-5601.

[91] S. Navalon, A. Dhakshinamoorthy, M. Alvaro, M. Antonietti, H. García, Active sites on graphene-based materials as metal-free catalysts, Chemical Society Reviews, 46 (2017) 4501-4529. 